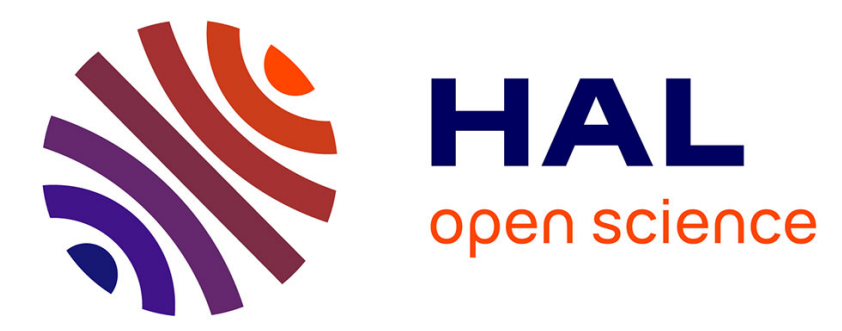

\title{
Recording of fast activity at the onset of partial seizures: Depth EEG vs. scalp EEG.
}

\author{
Délphine Cosandier-Rimélé, Fabrice Bartolomei, Isabelle Merlet, Patrick
}

Chauvel, Fabrice Wendling

\section{- To cite this version:}

Délphine Cosandier-Rimélé, Fabrice Bartolomei, Isabelle Merlet, Patrick Chauvel, Fabrice Wendling. Recording of fast activity at the onset of partial seizures: Depth EEG vs. scalp EEG.. NeuroImage, 2012, 59 (4), pp.3474-87. 10.1016/j.neuroimage.2011.11.045 . inserm-00664028

\section{HAL Id: inserm-00664028 https://www.hal.inserm.fr/inserm-00664028}

Submitted on 24 Feb 2012

HAL is a multi-disciplinary open access archive for the deposit and dissemination of scientific research documents, whether they are published or not. The documents may come from teaching and research institutions in France or abroad, or from public or private research centers.
L'archive ouverte pluridisciplinaire HAL, est destinée au dépôt et à la diffusion de documents scientifiques de niveau recherche, publiés ou non, émanant des établissements d'enseignement et de recherche français ou étrangers, des laboratoires publics ou privés. 
$8{ }^{1}$ Bernstein Center Freiburg, University of Freiburg, Freiburg, Germany

$9{ }^{2}$ INSERM, U751, Laboratoire de Neurophysiologie et Neuropsychologie, Marseille, France

$10 \quad{ }^{3}$ Université de la Méditerranée, Faculté de Médecine, Marseille, France

$11{ }^{4}$ CHU La Timone, Service de Neurophysiologie Clinique, AP-HM, Marseille, France

$12{ }^{5}$ INSERM, U642, Laboratoire Traitement du Signal et de l'Image, Rennes, France

$13 \quad{ }^{6}$ Université de Rennes 1, LTSI, Rennes, France

17 Correspondence:

18 Fabrice Wendling

19 LTSI - INSERM U642

20 Université de Rennes 1

21 Campus de Beaulieu, Bât. 22

2235042 Rennes Cedex, France

23 Email: fabrice.wendling@univ-rennes1.fr 


\section{Abstract}

2 Rapid discharges $(25-80 \mathrm{~Hz})$, a characteristic EEG pattern often recorded at seizure onset in 3 partial epilepsies, are often considered as electrophysiological signatures of the epileptogenic 4 zone. While the recording of rapid discharges from intracranial electrodes has long been 5 established, their observation from the scalp is challenging. The prevailing view is that rapid 6 discharges cannot be seen clearly (or at all) in scalp EEG because they have low signal-to-noise 7 ratio. To date, however, no studies have investigated the 'observability' of rapid discharges, i.e. 8 under what conditions and to what extent they can be visible in recorded EEG signals. Here, we 9 used a model-based approach to examine the impact of several factors (distance to sources, skull 10 conductivity, source area, source synchrony, and background activity) on the observability of 11 rapid discharges in simultaneously simulated depth EEG and scalp EEG signals. In our 12 simulations, the rapid discharge was clearly present in depth EEG signals but mostly almost not 13 visible in scalp EEG signals. We identified some of the factors that may limit the observability 14 of the rapid discharge on the scalp. Notably, surrounding background activity was found to be 15 the most critical factor. The findings are discussed in relation to the presurgical evaluation of 16 epilepsy.

\section{Keywords:}

19 Rapid discharges, observability, depth EEG, scalp EEG, computer simulations, epilepsy. 


\section{1. Introduction}

2 Seizure onset in partial epilepsy is often characterized by the appearance of a sustained low3 voltage fast activity (typically in the beta-gamma range, i.e. $25-80 \mathrm{~Hz}$ ), also referred to as a 4 'rapid discharge'. Rapid discharges are often considered as electrophysiological signatures of 5 the 'epileptogenic zone', i.e. the brain region(s) involved in the generation of seizures (Bancaud 6 et al., 1965; Fisher et al., 1992; Wendling et al., 2003; Bartolomei et al., 2008). Furthermore, 7 from a clinical viewpoint, surgical prognosis has been found to be related to the removal of 8 brain areas generating rapid discharges (Alarcón et al., 1995; Lee at al., 2000; Wetjen et al., 9 2009; Bartolomei et al., 2010). Investigations of rapid discharges are therefore of paramount importance in the presurgical evaluation of patients with drug-resistant partial epilepsy, as they could lead to better identification of the epileptogenic zone.

The recording of rapid discharges from intracranial (subdural and depth) electrodes is now well established, and several studies have been dedicated to the investigation of the spatial, spectral, and temporal features of rapid discharges in intracranial EEG signals (Allen et al., 1992; Fisher et al., 1992; Alarcón et al., 1995; Wendling et al., 2003). By contrast, the observation of rapid discharges in scalp EEG is challenging. Several studies have investigated the scalp EEG correlates of intracranial fast onset activities. Most reported that seizure onset is correlated with EEG attenuation ('flattening') or no detectable scalp EEG changes (Gastaut and Broughton, 1972; Pacia and Ebersole, 1997), and only very few have actually reported the presence of rapid discharges (Worrell et al., 2002).

The common view is that rapid discharges cannot be seen clearly (or at all) in scalp EEG because they suffer from a low signal-to-noise ratio and also are often obscured by muscle artifacts. To date, however, no studies have investigated the 'observability' of rapid discharges,

24 i.e. under what conditions and to what extent they can be visible in recorded EEG signals. The observability of any specific EEG activity depends on the complex interaction of several factors such as the spatiotemporal configuration of the sources, the distance from the recording

27 electrode to the sources, the background activity, and the electrical properties of the conductive 
1 media in the head. A thorough understanding of the biophysics of EEG measurements therefore

2 requires isolating the contribution of each of these factors. Such an investigation is hardly

3 possible to perform experimentally. However, it can be conducted with computer models, which

4 provide a unique opportunity to study the relative influence of individual parameters as well as

5 their interrelationships.

6 We recently proposed a neurophysiology-inspired computational model that allows for the

7 investigation of the relationship between the activation of brain sources and the corresponding

8 EEG signals (Cosandier-Rimélé et al., 2007, 2008). This model combines an accurate

9 description of the intracerebral sources (accounting for both the geometry of cortical dipole

10 layers and the neuronal activities) with a biophysically relevant description of the transmission

11 of source activities from the cerebral cortex to the recording electrodes. In previous studies, we

12 used the model to study the influence of source-related parameters (location, area, geometry,

13 and synchrony) on the properties of simulated signals during interictal spike activity, and

14 established quantitative relations between these parameters and the observability of spikes in

15 depth and scalp EEG signals (Cosandier-Rimélé et al., 2007, 2008).

16 In the present work, a similar model-based approach was used to investigate the impact of 17 different factors (source-to-sensor distance, skull conductivity, source area, source synchrony, 18 and background activity) on the contribution of rapid discharges to EEG signals. The objective 19 was to examine the conditions that facilitate or impair the recording of rapid discharges. To this 20 purpose, the EEG generation model has been adapted in order to produce fast activity as 21 observed at seizure onset in partial epilepsies. Then, the model was used to explore the specific 22 effects of each parameter on the observability of rapid discharges (as assessed by the 'energy 23 ratio' statistic) in simulated EEG signals. The investigation was conducted in simultaneously 24 simulated depth and scalp EEG signals, for comparative study of the sensitivity and specificity 25 of the two recording techniques. 


\section{2. Methods}

\subsection{Computational model of EEG signals}

4 Our computational, generative model of EEG signals combines a neurophysiology-based model 5 for the simulation of neuronal activity in the cerebral cortex with a biophysically relevant 6 description of the transmission of electric fields from the cerebral cortex to the recording 7 electrodes.

\subsubsection{Generation of neuronal activity}

10 Cortical neuronal activity was simulated using a population-level modeling approach that 11 describes the average activity of neural ensembles, without explicit representation of individual cell properties. Readers may refer to (Deco et al., 2008; Wendling and Chauvel, 2008) for recent reviews and detailed information on population-level models, also referred to as 'neural mass'

14 models.

In our model, a neuronal population consists of three interacting subpopulations: a subpopulation of excitatory pyramidal cells and two subpopulations of inhibitory interneurons mediating synaptic currents with either slow or fast synaptic kinetics (Fig. 1A). This model is a modified version of the model proposed in (Wendling et al., 2002, 2005), initially developed for hippocampus. This three-subpopulation model also complies with the neocortical cellular organization, as the neocortex is mainly composed of excitatory pyramidal neurons and inhibitory interneurons (Somogyi et al., 1998; Markram et al., 2004). In addition, there is experimental evidence for the presence of both slow and fast forms of inhibition in neocortex, which are mediated by two distinct subpopulations of interneurons (Benardo, 1994; Tamás et al., 2003). The intrinsic connectivity parameters (average number of synaptic contacts between pyramidal cells and inhibitory interneurons) have been changed according to anatomical data on neocortical circuits (Markram et al., 2004). Moreover, in the present work, several neuronal 
1 populations were considered and connected to each other (Jansen and Rit, 1995; Wendling et al.,

2 2000). All parameters and equations of the model are provided in the Appendix.

\section{$4 \quad 2.1 .2$ Calculation of electrical potentials}

5 Simulated EEG signals were calculated by solving the so-called 'EEG forward problem', i.e. by 6 computing the electric field potentials for a given source configuration in the head volume

7 conductor. Our spatio-temporal source model and potential calculations have been described in 8 details elsewhere (Cosandier-Rimélé et al., 2007, 2008). Main assumptions and principles are 9 summarized hereafter.

Source model - The EEG sources were assumed to be the cortical pyramidal neurons (i.e. activity in subcortical structures was not considered in the model). They were represented as dipole layers distributed over the neocortex surface. The convoluted anatomy of the neocortex

14 was accounted for by using a triangular mesh of the cortical surface, obtained from the segmentation of 3D resonance magnetic images. In this high resolution mesh (mean triangle area of $\sim 1 \mathrm{~mm}^{2}$ ), each mesh triangle was assumed to correspond to a distinct cortical neuronal population. The electrical contribution of each population was represented by a current dipole positioned at the barycenter of the corresponding mesh triangle and oriented perpendicular to its surface. The magnitude of the moment of each dipole was proportional to the area of the mesh triangle. Its time-course, which represents the time-varying dynamics of the associated

21 population, was provided by the output of the neuronal population model (summation of postsynaptic potentials), as described above.

24 Potential calculation - Electric potentials were calculated at both scalp electrodes (scalp EEG) 25 and depth-electrode contacts (depth EEG). Calculations were performed in the quasistatic 26 approximation, i.e. we assumed that the activity of sources projected instantaneously (i.e. with 27 no time delays) onto recording electrodes (Plonsey and Heppner, 1967). On the one hand, scalp 28 EEG measurements are very sensitive to the geometrical and electrical properties of the head 
1 tissues through which electric fields are transmitted. Scalp potential calculations were thus

2 performed in a realistically shaped head model obtained from the segmentation of 3D magnetic

3 resonance images. This model consisted of three electrically homogeneous compartments

4 representing the brain, the skull, and the scalp. The boundary element method (Hämäläinen and

5 Sarvas, 1989, Meijs et al., 1989) was used to compute the scalp potentials. On the other hand, in

6 intracerebral recordings, both sources and electrodes are located within the brain and therefore,

7 depth EEG measurements are mostly sensitive to the properties of the surrounding brain tissue.

8 In this particular case, forward calculations can be simplified by assuming the head is a

9 homogeneous brain region, i.e. omitting the skull and scalp compartments, and by considering it

10 as an infinite medium (Cosandier-Rimélé et al., 2007). The intracerebral potentials were thus

11 computed using the electrostatic potential formulation (Malmivuo and Plonsey, 1995).

\section{$13 \quad 2.1 .3$ Computer simulations}

14 In this study, we assumed a 'focal' organization of the epileptogenic zone, i.e. epileptic activity 15 was generated by only one neocortical region (Aubert et al., 2009). The source of epileptic 16 activity was simulated by using a set of contiguous triangles on the cortical mesh, or 'patch' 17 (Fig. 1C). Dipoles within the patch were assigned an epileptic time-course (fast activity), while 18 all other dipoles, outside the patch, were assigned a 'normal' time-course to simulate 19 surrounding background EEG activity. The two conditions (normal and epileptic) were obtained 20 from two different parameter settings (related to excitation and inhibition within a population) 21 in the neuronal population model (Fig. 1B). Specifically, the model generated a fast activity if 22 slow dendritic inhibition was decreased such that the populations of neurons became 'more 23 excitable', while keeping the capacity of generating fast inhibitory postsynaptic potentials, as 24 already shown in (Wendling et al., 2002, 2005). Model parameter values are provided in 25 Appendix (see Table 1).

26 From this extended cortical source model, the EEG signals were simulated simultaneously 27 on scalp electrodes as well as on depth-electrode contacts. Scalp potentials were computed at 32 28 positions on the scalp surface of the head model. They corresponded to a subset of 28 electrodes 
1 of the 10/10 system, and 4 additional subtemporal electrodes (FT9, FT10, TP9, and TP10).

2 Intracerebral potentials were computed at 10 contact positions along a virtual depth electrode

3 orthogonally placed in the epileptic source (electrode entry point in the 'middle' of the cortical

4 patch). The depth-electrode contacts were represented as points spaced at $3.5 \mathrm{~mm}$.

5

\section{$6 \quad 2.2$ Model parameter sensitivity analysis}

7 The observability of a given EEG activity depends on several factors that are related to the 8 source configuration and to volume conduction (i.e. conditions of the transmission of electric 9 fields from the sources to recording electrodes). In this work, we focused on three factors in the 10 former category (source area, source synchrony, and background activity), and two factors in 11 the latter category (source-to-sensor distance and skull conductivity). Five simulation scenarios were thus designed, each one addressing the specific influence of one of these factors on the observability of rapid discharges in depth and scalp EEG signals, comparatively. They are detailed hereafter, along with a description of the model parameter under investigation in each case.

Besides, the results of our sensitivity analysis may depend on the selected source location in the brain. Indeed, the neocortex surface is highly convoluted and the particular location, geometry, and 'orientation' of cortical sources with regard to the recording electrodes markedly impact the measured potential distributions (Gloor, 1985). To take account of the influence of these factors, and ensure we capture the general trends of the effects of investigated parameters, all our simulations were performed for ten different positions of the epileptic cortical patch (P1P10, Fig. 1D). All ten positions were arbitrarily lateralized in left hemisphere.

\subsubsection{Simulation scenario 1 - Effect of the source-to-sensor distance (parameter D)}

It is acknowledged that the distance of the source to the measuring sensor influences the EEG signal amplitude, since the potential falls off as a function of this distance. In this simulation scenario, the impact of source-to-sensor distance (parameter D) on the observability of rapid discharges was analyzed by comparing fast activities simulated at three recording sites, located 
1 at three different distances from the source: the source itself (i.e. within the epileptic cortical

2 patch), depth-electrode contacts, and scalp electrodes. The activity of the epileptic patch was

3 approximated by summing the fast onset activities generated by neuronal populations within the

4 patch, weighted by the area of their corresponding cortical mesh triangle. The distance between

5 a measurement sensor and the epileptic cortical patch was computed as the mean Euclidean

6 distance from the sensor to all dipoles within the patch. Additionally, to ensure we isolate the

7 influence of distance from volume conductor modeling effects (related to the geometry and

8 electrical properties of the head model), both intracerebral and scalp forward calculations were

9 performed in an infinite homogeneous volume conductor (in contrast with the other four

10 simulation scenarios, in which scalp forward calculations were performed using the realistic

11 head model). The conductivity of the medium was set to $0.33 \mathrm{~S} / \mathrm{m}$ (a commonly used value for

12 brain conductivity), and simulations were performed with a medium-sized $\left(\mathrm{S}=10 \mathrm{~cm}^{2}\right)$,

13 moderately synchronized (K=K2) epileptic patch and surrounding background activity $(\alpha=1)$.

\subsubsection{Simulation scenario 2 - Effect of the skull conductivity (parameter $\mathrm{R}$ )}

It is well known that the skull has a very low conductivity relative to the other head compartments. Because of its low conductivity, the skull strongly attenuates electric fields as they flow through it. However, there is no consensus on the skull conductivity value and a wide range of values have been reported (Rush and Driscoll, 1968; Oostendorp et al., 2000;

20 Gonçalves et al., 2003; Lai et al., 2005). The purpose of this simulation scenario was therefore 21 to investigate the impact of the skull conductivity value on the observability of rapid discharges in scalp EEG signals. Depth EEG signals were not considered here. Scalp potentials were computed using the realistic head model. The conductivities of the brain and the scalp were assumed to be identical and were set to $0.33 \mathrm{~S} / \mathrm{m}$. The conductivity of the skull was expressed as

25 a fraction of the conductivity of the brain, and we defined a parameter $\mathrm{R}$ as the brain-to-skull 26 conductivity ratio. Four values of $\mathrm{R}$ were used for the simulations: 10, 20, 40 (the 'standard' 27 value, used in the other four simulation scenarios) and 80 , in accordance with the values 28 reported in the above-cited studies. In addition, to isolate the influence of conductivities from 
1 the geometrical properties of the head model, we also considered $\mathrm{R}=1$ (i.e. a head model

2 comprising three compartments with identical conductivities) as an 'initial' condition.

3 Simulations were performed with a medium-sized $\left(\mathrm{S}=10 \mathrm{~cm}^{2}\right)$, moderately synchronized

$4(\mathrm{~K}=\mathrm{K} 2)$ epileptic cortical patch and surrounding background activity $(\alpha=1)$.

5

$6 \quad$ 2.2.3 Simulation scenario 3 - Effect of the source synchrony (parameter K)

7 The synchronization of neuronal activity within the epileptic source is a crucial determinant of

8 the recorded EEG patterns. In our model, the source synchrony 'level' is controlled by using the

9 degree of coupling between the neuronal populations within the source. In the neuronal 10 population model, the connection from a given population $i$ to a population $j$ is characterized by 11 a parameter $\mathrm{K}_{i j}$ that represents the coupling strength (Jansen and Rit, 1995; Wendling et al., 12 2000). Based on recent findings on the coupling of neuronal fast activities (Wendling et al., 13 2010), we assumed a 'star-like' network with unidirectional coupling (i.e. all populations are 14 connected to a center population), and all parameters $\mathrm{K}_{i j}$ were set to a same value $\mathrm{K}$. In this 15 simplistic connectivity model, the source synchrony level can be more easily controlled 16 compared to situations where random bi-directional coupling are present in the network. Its 17 influence can indeed be analyzed just by varying parameter K. Specifically, we used four 18 different values of $\mathrm{K}$, to account for a null $(\mathrm{K}=0)$, low $(\mathrm{K} 1)$, medium (K2), and high (K3) degree 19 of coupling between the neuronal populations. Both depth EEG and scalp EEG signals were 20 simulated, using a medium-sized $\left(\mathrm{S}=10 \mathrm{~cm}^{2}\right)$ epileptic patch and surrounding background 21 activity $(\alpha=1)$.

\subsubsection{Simulation scenario 4 - Effect of the source area (parameter S)}

Determining the size of cortical sources of recorded epileptic EEG activities is a key issue in the presurgical evaluation of epilepsy. To study the relationship between the spatial extent of the source and the observability of rapid discharges in simulated EEG signals, we considered an

27 epileptic cortical patch with a varying surface area S. The surface area of a cortical patch was 28 defined as the sum of the surface areas of elementary mesh triangles included within the patch. 
1 In simulations, the patch surface area $\mathrm{S}$ was varied from 1 to $30 \mathrm{~cm}^{2}$, by steps of $1 \mathrm{~cm}^{2}$ (Fig. 1E),

2 and both depth and scalp EEG signals were simulated. The two other source-related parameters

3 were kept constant (source synchrony level: $\mathrm{K}=\mathrm{K} 2$, and background activity level: $\alpha=1$ ).

4

$5 \quad$ 2.2.5 Simulation scenario 5 - Effect of the background activity (parameter $\alpha$ )

6 In general, it is recognized that locally generated EEG activities tend to be overshadowed by the

7 background activity that is concurrently generated throughout the brain. In our model, the

8 simulated EEG results from the superposition of the potentials produced by the epileptic cortical

9 patch (generating fast activity) and those produced by the rest of the cortex (generating

10 background activity). The purpose of this simulation scenario was to examine the impact of the

11 amplitude of the background activity on the observability of rapid discharges in EEG signals.

12 To do so, we introduced a parameter $\alpha$ that 'weights' the relative contribution of the background

13 activity to the simulated EEG. Depth and scalp EEG signals were simulated for all $\alpha$ values

14 between 1 ('control' condition) and 0 (no background), with a step of 0.1 . Simulations were

15 performed with a medium-sized $\left(\mathrm{S}=10 \mathrm{~cm}^{2}\right)$, moderately synchronized $(\mathrm{K}=\mathrm{K} 2)$ epileptic patch.

\section{$17 \quad 2.3$ Quantification of rapid discharges}

18 The salient feature of rapid discharges is a redistribution of the energy of EEG signals from low

19 frequencies (in the delta-theta-alpha range) toward higher frequencies (typically, in the betagamma range) (Bartolomei et al., 2008). In our model, the frequency range of fast activity is 20-

$2125 \mathrm{~Hz}$ (in the high beta band), in contrast with frequencies of the background activity which are mostly $<10 \mathrm{~Hz}$ (in delta and theta bands) (Fig. 1B). To study and illustrate the frequency content of simulated EEG signals, we used the power spectral density (PSD, periodogram method). Furthermore, we assessed the observability of rapid discharges in EEG signals by computing the ratio of the energy of the EEG in two frequency bands: 0.1 to $8 \mathrm{~Hz}$ (lowfrequency energy, LE) and 18 to $30 \mathrm{~Hz}$ (high-frequency energy, HE). This 'energy ratio' (ER), previously introduced in (Bartolomei et al., 2008), quantifies how much the rapid discharge 
1 (HE) 'emerges' from the background (LE). It is computed from the signal spectral density $\Gamma$

2 (squared modulus of its Fourier transform):

$$
\mathrm{ER}=\mathrm{HE} / \mathrm{LE}, \text { with } \mathrm{LE}=\int_{f=0.1}^{f=8} \Gamma(f) d f \text { and } \mathrm{HE}=\int_{f=18}^{f=30} \Gamma(f) d f
$$

In practice, the ER values were computed as functions of time, over a sliding analysis window. We denote by ER[n] the values computed at discrete time index $\mathrm{n}$ of the window position. The window length was set to $4 \mathrm{~s}$, and the sliding step to $0.25 \mathrm{~s}$. The duration of the simulations was 30 s. The ER values were computed over the whole simulation duration $(\mathrm{N}=105$ values). Then, for each simulated EEG signal $s_{\mathrm{i}}$, we computed the mean ER $\left(\mathrm{mER}_{\mathrm{i}}\right)$ by averaging the values over time:

$$
\operatorname{mER}_{i}=\frac{1}{N} \sum_{n=1}^{N} \operatorname{ER}_{i}[\mathrm{n}]
$$

11 where i denotes the considered scalp electrode or depth-electrode contact.

Furthermore, in order to compare the results at the different patch locations, the $\mathrm{mER}_{\mathrm{i}}$ values were normalized between 0 (no rapid discharge) and 1 ('maximal' rapid discharge). For each patch location, $\mathrm{mER}_{\mathrm{i}}$ values were divided by the maximum value obtained in all simulations. In 9 out of 10 cases, this maximum value was obtained in simulation scenario 1 , from the signal simulated at the level of the epileptic cortical source. In one case (P5), it was obtained in simulation scenario 5 , in the absence of background activity $(\alpha=0)$. In the sequel, normalized $m E R_{i}$ values are simply denoted by ' $m E R$ values'.

\subsection{Statistical analysis}

21 Statistical analysis was performed to assess the effect of the investigated factors on the 22 observability of rapid discharges in simulated EEG signals. For each patch location, the mER 23 values were computed for each simulated depth and scalp EEG signal. The analysis then

24 focused on the EEG signals from the depth-electrode contact and the scalp electrode that are closest to the epileptic cortical patch. We used box plots to compare the statistical distributions of the mER values, as computed for different parameter settings. On each box, the central mark 
1 is the median, the edges of the box are the 25 th and 75 th percentiles, the length of the whiskers

2 is 1.5 times the interquartile range, and any data point beyond the whiskers (outlier) is plotted

3 with a star. Furthermore, to assess the statistical significance of the differences in mER, we used

4 nonparametric Wilcoxon rank sum tests, with a significance level of $1 \%$.

5

$6 \quad$ 3. Results

7

$8 \quad 3.1$ Examples of real rapid discharges

9 Before describing the results of the computer simulations, we present examples of real rapid 10 discharges recorded in two epileptic patients with drug-resistant epilepsy, during the transition 11 from normal to seizure activity. Patient \#1 had focal cortical dysplasia located in the right lateral occipital cortex and has been previously studies in a series of patients with focal epileptogenic lesion (patient \#6 in (Aubert et al. 2009)). Patient \#2 had cryptogenic, left frontal lobe epilepsy.

14 Patient \#1 illustrates the case of a rapid discharge that is visible both in depth EEG and in scalp EEG recordings (Figs. 2A-B), while patient \#2 illustrates a case of a rapid discharge that is clearly present in depth EEG recordings but not visible in scalp EEG recordings (Figs. 2C-D). In both cases, the rapid discharge is characterized by a narrowband activity (quasi-sinusoidal signal), with a frequency between 15 and $20 \mathrm{~Hz}$ in patient \#1 and between 25 and $30 \mathrm{~Hz}$ in patient \#2. When visible on the scalp (patient \#1), the rapid discharge is highly contaminated with low-frequency $(<10 \mathrm{~Hz})$ background activity. Simulated rapid discharges resemble real ones in terms of morphology and spectral content, in both EEG modalities, as seen in simulation examples shown below.

\subsection{Simulation scenario 1 - Effect of the source-to-sensor distance}

Results about the influence of the source-to-sensor distance (parameter D) are illustrated in Fig.

3. The observability of the rapid discharge greatly deteriorated as the recording site moved away from the epileptic patch, as shown by a significant decrease in mER (Fig. 3A). The average 
$1 \mathrm{mER}$ was $1.0 \pm 0.0($ mean \pm standard deviation $)$ at the cortical sources, $32.39 \times 10^{-3} \pm 16.51 \times 10^{-3}$

2 at depth-electrode contacts (with D values ranging from 9 to $10 \mathrm{~mm}$ ), and $1.40 \times 10^{-3} \pm 1.08 \times 10^{-3}$

3 at scalp electrodes (D from 22.3 to $31.7 \mathrm{~mm}$ ). Visual inspection of simulated signals and their

4 spectra indicated that increasing the distance both decreased the high-frequency energy of the

5 simulated signals and increased the low-frequency energy (Figs. 3B-E). However, it appeared

6 that the increase in LE was much more important than the decrease in HE. Indeed, while the

7 rapid discharge was accompanied only by slight background activity in depth EEG signals, the

8 two activities were 'blended' in scalp EEG signals and the rapid discharge was little (or not)

$9 \quad$ visible in this case (Fig. 3E).

\section{$11 \quad 3.3$ Simulation scenario 2 - Effect of the skull conductivity}

Fig. 4 illustrates the effect of varying the brain-to-skull conductivity ratio (parameter R). The mER was progressively reduced with increasing R (i.e. decreasing skull conductivity) (Fig. 4A). However, there were no statistically significant differences in mER between the five tested values of $\mathrm{R}$. In addition, the $\mathrm{mER}$ values were already very low $\left(<3 \times 10^{-3}\right)$ for $\mathrm{R}=1$ (i.e. when the brain, skull, and scalp had an equal conductivity). Visual inspection of simulated signals and their spectra confirmed these observations and further revealed a marked attenuation of the amplitude of the scalp EEG with increasing R (Fig. 4B).

\subsection{Simulation scenario 3 - Effect of the source synchrony}

Fig. 5 illustrates the effect of increasing the degree of coupling (parameter K) between the neuronal populations within the epileptic patch. Fast activities were asynchronous for a null coupling and became more synchronized as $\mathrm{K}$ increased. Thereby, the level of synchrony of the source (as assessed by calculating the correlation coefficients (nonlinear regression coefficients $\mathrm{h}^{2}$ (Pijn and Lopes da Silva, 1993; Wendling et al., 2001)) between population pairs within the patch) increased with increasing K (Figs. 5A-B). At the level of the electrodes, the observability 
1 coupling $(\mathrm{K}=0)$, the mER values were very low in both depth and scalp EEG signals. In depth

2 EEG signals, the mER was drastically increased between $\mathrm{K}=0$ and $\mathrm{K}=\mathrm{K} 1$ (the average mER was

3 multiplied by $\sim 16$ ), and then the amount of increase became progressively smaller (Fig. 5D). In

4 scalp EEG, there was a more gradual increase in mER with increasing $\mathrm{K}$ (Fig. 5F). In both

5 modalities, however, the difference in $\mathrm{mER}$ was no longer significant between $\mathrm{K}=\mathrm{K} 2$ and $\mathrm{K}=\mathrm{K} 3$.

6 The range of variation in mER was much wider in depth EEG (average mER between $0.59 \times 10^{-3}$

$7 \pm 0.29 \times 10^{-3}$ for $\mathrm{K}=0$ and $51.41 \times 10^{-3} \pm 24.73 \times 10^{-3}$ for $\mathrm{K}=\mathrm{K} 3$ ) than in scalp EEG (between

$80.25 \times 10^{-3} \pm 0.02 \times 10^{-3}$ and $\left.0.95 \times 10^{-3} \pm 0.44 \times 10^{-3}\right)$. Visual inspection of simulated signals and

9 their spectra showed that increasing the neuronal coupling within the epileptic patch resulted in

10 a marked increase in high-frequency energy of the simulated EEG. In contrast with depth EEG

11 signals where the rapid discharge was very visible from $\mathrm{K}=\mathrm{K} 1$ onwards (Fig. 5C), in scalp EEG

12 signals, the rapid discharge mostly hardly emerged from background activity for $\mathrm{K}=\mathrm{K} 3$ (Fig.

$135 \mathrm{E})$.

14

$15 \quad 3.5$ Simulation scenario $4-$ Effect of the source area

16 Results about the effect of extending the source surface area (parameter S) are shown in Fig. 6.

17 In both depth and scalp EEG signals, the observability of the rapid discharge was significantly

18 improved as the epileptic patch got larger. However, the relation between the mER and $\mathrm{S}$

19 appeared to be very different in the two modalities. In depth EEG, the amount of increase in

20 mER was greatest for small patch surface areas and rapidly leveled off (Fig. 6A). Indeed, for 7

21 out of the 10 patch locations, the mER reached a 'plateau' level at surface areas between 5 and

$2210 \mathrm{~cm}^{2}$. In scalp EEG, by contrast, there was a more gradual increase in mER with increasing $\mathrm{S}$,

23 although with some variability in the slope of the relation between patch locations (Fig. 6B). In

24 addition, the range of variation in mER was narrower in scalp EEG (average mER between

$250.27 \times 10^{-3} \pm 0.02 \times 10^{-3}$ at $\mathrm{S}=1 \mathrm{~cm}^{2}$ and $2.26 \times 10^{-3} \pm 1.27 \times 10^{-3}$ at $\mathrm{S}=30 \mathrm{~cm}^{2}$ ) than in depth EEG

26 (between $1.21 \times 10^{-3} \pm 1.27 \times 10^{-3}$ and $77.23 \times 10^{-3} \pm 62.43 \times 10^{-3}$ ). Visual inspection of simulated

27 signals and their spectra indicated that the rapid discharge was clearly visible for very small

28 surface areas of the epileptic patch $\left(<3 \mathrm{~cm}^{2}\right)$ in depth EEG signals (Fig. 6C) whereas, in scalp 
1 EEG signals, it emerged from background activity only for large surface areas $\left(>10 \mathrm{~cm}^{2}\right)$ (Fig.

2 6D). For one epileptic patch (P4), the rapid discharge was never visible on the scalp, even for $3 \mathrm{~S}=30 \mathrm{~cm}^{2}$.

4

\section{$5 \quad 3.6$ Simulation scenario 5 - Effect of the background activity}

6 Fig. 7 shows the results about the effect of varying the background activity level (parameter $\alpha$ ).

7 In both EEG modalities, the observability of the rapid discharge significantly deteriorated with 8 increasing background level. In the absence of background $(\alpha=0)$, the mER values were very 9 high $(>0.85)$ and comparable for depth and scalp EEG signals. In the presence of background $10(\alpha>0)$, on the other hand, the relation between the mER and $\alpha$ was found to be very different in 11 the two recording techniques. In scalp EEG, there was a drastic reduction in mER for small $\alpha$ values (Fig. 7B). Indeed, the average mER was divided by $\sim 100$ between $\alpha=0$ and $\alpha=0.2$, and by $\sim 500$ between $\alpha=0$ and $\alpha=0.5$. By contrast, in depth EEG, the mER decreased more gradually between $\alpha=0$ and $\alpha=1$, for all ten patch locations (Fig. 7A). In addition, the mER values remained much higher for $\alpha=1$ in depth EEG $\left(32.39 \times 10^{-3} \pm 16.51 \times 10^{-3}\right)$ than in scalp EEG $\left(0.65 \times 10^{-3} \pm 0.26 \times 10^{-3}\right)$. Visual inspection of simulated signals and their spectra showed that, in scalp EEG, the rapid discharge rapidly 'blended' with background activity and it became less visible for middle values of $\alpha$ already (Fig. 7D), while in depth EEG, the rapid discharge was very clearly visible for all $\alpha$ values (Fig. 7C).

\section{Discussion}

To our knowledge, this study is the first that uses computer simulations to quantitatively address the influence of different factors on the observability of rapid discharges at seizure onset, in depth EEG and scalp EEG signals comparatively. The main findings are discussed below, along with the limitations of the present study and suggestions for future research.

\subsection{Low observability of rapid discharges in scalp EEG}


1 A first general result is that the mER values obtained in scalp EEG were significantly lower

2 than those in depth EEG (they differed by one or two orders of magnitude). Visual inspection of

3 simulated signals and their spectra indicated that in many cases, the rapid discharge could not be

4 seen clearly (or at all) in scalp EEG, while simultaneously it was very visible in depth EEG.

5 Therefore, our model results are in agreement with prevailing views and further suggest that the

6 situations where favorable conditions are met for observing rapid discharges in scalp EEG

7 remain much more specific as compared with depth EEG. In addition, it should be mentioned

8 that our computer simulations were conducted in a well-controlled framework, unlike real scalp

9 EEG recordings where signals are also contaminated by artifacts (eye movements, muscle, etc.)

10 that further hamper the observation of rapid discharges. Our results may therefore overestimate

11 the actual observability of rapid discharges in scalp EEG signals.

\section{$13 \quad 4.2$ Minor contribution of the attenuating effect of the skull}

14 When examining the attenuating effect of the skull (simulation scenario 2), our results indicated 15 that, as expected, decreasing skull conductivity reduced the observability of the rapid discharge 16 in simulated scalp EEG signals. However, the reduction in mER was very small and not 17 statistically significant. Moreover, very low mER values were also obtained with a conductive 18 skull. Altogether, these findings suggest that the low skull conductivity is only a minor factor in 19 the lack of observable rapid discharges in scalp EEG, which contradicts the prevailing view on 20 the importance of this factor.

21 Besides, it should be emphasized that the lack of observable scalp rapid discharges is only 22 due to voltage attenuation, and that the skull does not filter out high-frequency components of 23 brain activity, as it is sometimes said. Indeed, it has long been established that the quasistatic 24 approximation holds for the EEG, i.e. there is no time-lag between electrical potentials recorded at any point in the head and the underlying source activity (Plonsey and Heppner, 1967). Under these conditions, the transfer coefficients from any source in the brain to any measuring sensor

27 (the so-called 'EEG lead field') depend only on the volume conductor properties (geometry and 28 conductivities of the head tissues) and on the positions and orientations of the sources with 
1 respect to sensors. Thus, the spectral content of the neuronal activity is not altered during its

2 transmission from the cerebral cortex to the scalp, and the skull hampers the recording of rapid

3 discharges only because its low conductivity attenuates an already small activity (Gotman,

4 2010).

5

\section{$6 \quad 4.3$ Major impact of background activity and distance}

7 In our simulations, the level of background activity appeared to be the most critical factor

8 impeding the observability of rapid discharges. For both EEG modalities, the most conspicuous

9 variations in mER were observed in simulation scenario 5, where we found that $\mathrm{mER}$ values

10 were very high (close to 1) in the absence of background activity and were markedly reduced

11 with increasing background level. The impact of background activity appeared to be particularly

12 dramatic in scalp EEG signals, where a slight increase in background level resulted in a drastic

13 decrease in mER. While the role of background activity in EEG analysis and interpretation has

14 been recognized, these findings further highlight the crucial importance of this factor.

15 As expected, results indicated that the depth EEG was less susceptible to background 16 activity than the scalp EEG. Depth electrodes can be in direct contact with or in close vicinity to

17 some sources, which provides higher sensitivity but smaller volume of sensitivity to the depth

18 EEG recording technique. An advantage of a more restricted volume of sensitivity is that it

19 reduces the amount of surrounding background activity captured by the electrodes. Depth EEG

20 therefore enables a more reliable measure of rapid discharges, provided the electrodes are

21 accurately positioned in the involved brain region(s). In contrast, scalp electrodes remain remote

22 from brain sources. Their volume of sensitivity is thus very large and they record 'mixtures' of source activities from the entire brain. Rapid discharges are difficult to observe in scalp EEG

24 because they originate from a limited brain area and they are overshadowed by the background 25 activity that is concurrently generated throughout the brain.

26 Besides, it should be mentioned that in our model, the background EEG was produced by 27 concurrently active neuronal populations distributed over the whole cortical surface, and that 28 these populations were independent from each other (non-coupled neuronal population models). 
1 This is unlike real background EEG, as brain connectivity (structural, functional, and effective)

2 induces correlations in activity between different brain regions (Sporns, 2010). The presence of

3 correlated activity would probably increase the relative contribution of the background to the

4 recorded EEG signal and thereby, even further overshadow the local rapid discharges. Our

5 results may therefore overestimate the actual observability of rapid discharges.

6 As far as the source-to-sensor distance is concerned, the results in simulation scenario 1

7 indicated a strong impact of this factor, as shown by the significant decrease in mER with

8 increasing distance. Interestingly, we found a greater effect on low-frequency energy (LE) than

9 on high-frequency energy (HE) of the simulated EEG, i.e. a much more important increase in

10 LE compared with the decrease in HE. This finding suggests that distance influences the

11 observation of rapid discharges in recorded signals through its impact on the volume of

12 sensitivity of the electrode which, in turn, affects the amount of background activity captured by

13 the electrode. This view is further supported by results in simulation scenario 5 , which indicated

14 that in the absence of background activity, the mER values were similar at the three considered

15 recording sites (cortical source, depth electrode, and scalp electrode). One likely explanation of

16 this finding is the linear relationship that exists between the amplitude of a measured potential

17 and the inverse of the distance between the electrode and a dipole layer (Cosandier-Rimélé et al.,

18 2007). Indeed, we previously showed that potential attenuation in the dipole layer model was

19 inversely proportional to source-to-sensor distance. Under these conditions, changing the

20 distance is therefore roughly equivalent to multiplying the EEG voltage by a weighting

21 coefficient. Given the definition of the mER quantity (a ratio of energies), it is insensitive to

22 such voltage 'scaling'.

$24 \quad 4.4$ Influence of the source area and source synchrony

25 Our results (simulation scenarios 3 and 4) showed that extension of the surface area of the 26 epileptic source and strengthening of neuronal coupling within the source were associated with 27 improved observability of rapid discharges in simulated EEG signals. These findings are 28 consistent with the well-known fact that only neuronal activities that are synchronous over 
1 rather large cortical areas yield potentials that can be recorded in EEG, and most particularly in

2 scalp EEG (Abraham and Ajmone-Marsan, 1958; DeLucchi et al., 1962; Cooper et al., 1965;

3 Tao et al., 2005, 2007; Cosandier-Rimélé et al., 2007, 2008). Interestingly, it has been suggested

4 that rapid discharges at seizure onset may originate from small brain areas (Tao et al., 2007) and

5 may be transiently desynchronized (Wendling et al., 2003), two conditions that would

6 contribute to make their recording from the scalp less likely. As expected, our simulation results

7 revealed a high sensitivity of the depth EEG, in which rapid discharges could be observed from

8 smaller cortical areas and lower synchrony levels as compared to the scalp EEG. However,

9 results in simulation scenario 4 also showed that the observability of rapid discharges in depth

10 EEG signals was not much improved when increasing the source area beyond $5-10 \mathrm{~cm}^{2}$, which

11 could demonstrate the spatial restriction of the volume of sensitivity of depth electrodes. As far

12 as scalp EEG signals are concerned, our results showed that the impact of source area and

13 source synchrony was relatively limited, as indicated by narrow ranges of variation in mER

14 when compared to depth EEG signals. It is noteworthy, however, that in our simulations, rapid

15 discharges became visible in scalp EEG signals for extended source area (simulation scenario 4).

16 The results in simulation scenario 3, on the other hand, showed that enhanced source synchrony

17 was not able to fully compensate for the inherent 'shadowing effect' of the background activity.

18 Overall, our simulation results are thus in agreement with previous clinical observations

19 indicating that rapid discharges are often not visible at seizure onset in scalp EEG recordings.

20 Strikingly, however, several recent studies demonstrated that fast activities $(>40 \mathrm{~Hz})$ can be

21 readily observed from the scalp (Kobayashi et al., 2004, 2009; Wu et al., 2008; Andrade-

22 Valenca et al., 2011). To some extent, these findings contrast with those reported in our study.

23 The most likely explanation for this discrepancy is the difference in the spatial configuration of

24 sources. Indeed, several studies reported on fast activities recorded in scalp EEG in patients

25 with severe types of epileptic seizures, such as epileptic spasms (Kobayashi et al., 2004; Wu et

26 al., 2008) and tonic seizures (Kobayashi et al., 2009), which generally reflect bilateral

27 widespread brain generators. A broader distribution of fast activities would contribute to make

28 them more visible on scalp recordings. This is in agreement with our finding that rapid 
1 discharges became more visible as the epileptic patch got larger (simulation scenario 4).

2 Interestingly, diffuse amplitude attenuation ('flattening') also may occur during these types of

3 seizures. This may relate to the situation where the level of surrounding background activity

4 was decreased in our simulations, a situation that was shown to improve the observability of

5 rapid discharges (simulation scenario 5). In addition, very recently, a study demonstrated that

6 fast activities can also be recorded in scalp EEG in patients with focal epilepsy (Andrade-

7 Valenca et al., 2011), a context that is more similar to that of our study. However, the authors

8 suggested that the patients presented a 'network' organization of the epileptogenic zone, i.e.

9 epileptic activity was generated by multiple brain regions. The synchronous activation of these

10 regions would broaden the distribution of fast activities and thereby, contribute to improve their

11 observability on scalp EEG. It is noteworthy that in our study, the patient with scalp-recorded

12 rapid discharges (patient \#1) presented an epileptogenic zone that included at least the temporo-

13 occipital junction and the mesial temporal cortex (entorhinal cortex). Future studies with

14 simulations using multiple epileptic sources are required to specifically address the issue of the

15 observability of rapid discharges in the context of a network organization of the epileptogenic

16 zone.

17 Besides, a comment should be made about the reduction in EEG amplitude that may occur at seizure onset. Although frequent, the 'low-voltage' aspect is not a constant feature of rapid discharges, suggesting at least two different situations of altered synchronization. First, most of

20 the neuronal populations within the epileptic source would generate synchronous fast activity as

21 a result of 'organized connectivity', as chosen in this study for the sake of simplicity. In this

22 case, resulting EEG signals are characterized by an increase of amplitude, as verified in our

23 simulations (Fig. 5). Second, in an alternative situation, each population within the patch would

24 generate a fast activity but independently from the others, as a result of random connectivity

25 patterns (in which the synchronization level is known to be more difficult to control and

26 measure (Wendling et al., 2010)). In this situation, it is expected that the low temporal

27 correlation among individual activities would lead to a decrease of amplitude in EEG signals.

28 As mentioned above, in this study, we chose a simple connectivity model, in order to examine 
1 the relation of a well-controlled and measurable synchronization level within the epileptic patch

2 to the presence of rapid discharges in resulting EEG signals. Dedicated studies are still to be

3 performed to analyze more specifically the influence of connectivity patterns on the

4 observability of rapid discharges. Nevertheless, it is acknowledged that the present connectivity

5 model, with induced increase in signal amplitude, increases the relative contribution of rapid

6 discharges in the mixture with background activity, and our results thus probably overestimate

7 the actual observability of rapid discharges in EEG signals.

\section{$9 \quad 4.5$ Impact of the source topology}

10 Results presented in this study were obtained from ten different positions of the epileptic

11 cortical source. All positions were arbitrarily distributed over the lateral cortical surface, in order to obtain rather similar signal-to-noise ratio levels in scalp EEG signals. Here, the objective was not to exhaustively investigate the influence of source location on the observability of rapid discharges, but rather to account for variability in mER values due to variations in cortical surface geometry depending on location. We acknowledge that the location of the cortical source is a crucial determinant of the observability of scalp EEG patterns in general, and of rapid discharges in particular. This issue was not addressed specifically in this work and would require a dedicated study to examine in details the impact of geometrical properties of the cortical source such as its position and 'net' orientation relative to the recording electrodes and to quantify the effects of gyral vs. sulcal activity.

\subsection{Neuronal fast activity}

23 Finally, it is noteworthy that the proposed neural mass model of a cortical population generates

24 'fast' activity at $20-25 \mathrm{~Hz}$. We showed that simulated fast activities are close to some real 25 patterns recorded at seizure onset in patients with partial neocortical epilepsy (Fig. 2). However, 26 rapid discharges with frequencies $>40 \mathrm{~Hz}$ are commonly recorded in the neocortex (Allen et al., 27 1992; Fisher et al., 1992; Traub et al., 2001; Wendling et al., 2003). It was not possible to 
1 generate, from our neuronal population model, such high-frequency activity, which indicates

2 that the model is incomplete. The mechanisms underlying the generation of high-frequency

3 oscillations (HFOs, 80-500 Hz) - either during interictal/preictal periods (Jacobs et al., 2008,

4 2009; Gotman, 2010) or at seizure onset (Traub et al., 2001) - remain elusive, and several

5 theories have been proposed (Bartos et al., 2007; Traub et al., 2010; see also (Bragin et al.,

6 2010) for a review). One theory involves the mutual inhibition in networks of interconnected

7 inhibitory interneurons (Whittington et al., 1995, 2000; Gnatkovsky et al., 2008). Recently,

8 neural mass models have been proposed which include mutual inhibition (inhibitory

9 connections among fast inhibitory interneurons) (Molaee-Ardekani et al., 2010; Ursino et al.,

10 2010). They have been shown to produce higher frequency activity, up to $100 \mathrm{~Hz}$ in (Molaee-

11 Ardekani et al., 2010). Using such a model of neuronal activity would help improve the realism

12 of our simulations. However, we would expect similar results from the model parameter

13 sensitivity analysis since results do not depend on the frequency of the discharge, but they

14 depend on the source configuration ('local' epileptic vs. background activity) and on the way

15 activities generated at the level of neuronal populations 'mix' together to produce the signals

16 that are recorded by the electrodes.

\subsection{Conclusion}

Rapid discharges observed at the onset of partial seizures are often considered as a hallmark of epileptogenic areas (Bancaud et al., 1965; Fisher et al., 1992; Wendling et al., 2003; Bartolomei et al., 2008). In addition, high-frequency oscillations (ripples, fast ripples) observed during interictal periods were also reported as potential biomarkers of the epileptogenic tissue (Jacobs et al., 2008; Gotman, 2010; Demont-Guignard et al., 2011). Therefore, given the increasing importance, in clinical epileptology, of activities within the beta/gamma bands (and beyond), it is essential to understand how these fast activities as generated at the level of cortical sources do reflect at the level of EEG signals, either locally (depth EEG) or globally (scalp EEG). This study constitutes a first step in this direction. However, it should be reminded that the results reported in the present study are only based on computer simulations. A second step would thus 
1 be to further validate these model-based results using clinical data. In order to perform this

2 experimental validation, simultaneously-recorded depth and scalp EEG data are required. This

3 is not a trivial issue for two reasons, at least. First, in practice, an appropriate scalp electrode

4 coverage is difficult to achieve, particularly in regions where intracerebral electrodes are

5 inserted. Second, due to the skull perforation at each intracerebral electrode location, the skull

6 integrity is modified. Nevertheless, although 'ideal' recording conditions can hardly be met in

7 patients, some parameters like the source surface area and source synchrony may be

8 investigated, as reported in (Tao et al., 2005, 2007). Some other parameters, like the level of

9 surrounding background activity (which, in our simulations, appears to be a critical factor in the

10 observability of rapid discharges in recorded EEG signals), are however less directly accessible

11 experimentally.

12

13 Acknowledgement

14 This work was funded in part by the French National Institute of Health and Medical Research 15 (Inserm).

\section{Appendix. Neuronal population model}

18 A cortical neuronal population is assumed to be composed of three interacting subpopulations of neurons: the excitatory pyramidal cells, inhibitory interneurons with slow synaptic kinetics, and inhibitory with fast synaptic kinetics (Fig. 1A). The structure of the population model is similar to that proposed in (Wendling et al., 2002). According to the neural mass approach, each neuronal subpopulation is described by its average membrane potential $(v)$ and mean firing rate $(r)$. A linear operator $h$ transforms $r$ into $v$ (describing the action of the synapses), while a static nonlinear operator $S$ relates $v$ to $r$ (modeling the action of the soma of neurons). The first operator is represented by a second-order lowpass filter, with an impulse response given by $h(\mathrm{t})=\mathrm{Wwte} \mathrm{e}^{-\mathrm{wt}} . \mathrm{u}(\mathrm{t})$, where parameters $\mathrm{W}$ and $\mathrm{w}$ represent the maximum amplitude and rate 
1 We used three different impulse responses, $h_{\mathrm{A}}$ (parameterized by A and a), $h_{\mathrm{B}}\left(\mathrm{B}\right.$ and $\mathrm{b}$ ), and $h_{\mathrm{G}}$

2 ( $\mathrm{G}$ and $\mathrm{g}$ ), to model the excitatory, slow inhibitory, and fast inhibitory synapses, respectively.

3 These values are given in Table 1 . The second operator is represented by a sigmoid function $S$

4 that mimics threshold and saturation effects taking place at the soma (see in (Jansen and Rit,

5 1995; Wendling et al., 2000) for details). Synaptic interactions between the neuronal

6 subpopulations are characterized by seven connectivity constants $\left(\mathrm{C}_{1}\right.$ to $\left.\mathrm{C}_{7}\right)$ that account for the

7 average numbers of synaptic contacts (see Table 2). In addition, the nonspecific influence from

8 neighboring or distant populations is represented by a Gaussian input noise $p$ (mean: 90,

9 standard deviation: 30) that globally represents the average density of incoming action

10 potentials.

11 Furthermore, in this work, several neuronal population models were considered and connected

12 to each other. Inter-population coupling was modeled by excitatory connections between

13 pyramidal cell subpopulations (Jansen and Rit, 1995; Wendling et al., 2000). The connection

14 from a given population $i$ to a population $j$ is characterized by a gain constant $\mathrm{K}_{\mathrm{ij}}$ that defines the

15 coupling strength, and a linear operator $h_{\mathrm{d}}$ (chosen to be similar to $h_{\mathrm{A}}$, for simplicity) that

16 models the delay associated with the connection. Consequently, the neuronal population

17 network model is described by a set of ten ordinary differential equations per population, i.e.

18 eight equations to describe the intra-population behavior (Wendling et al., 2002, 2005) and two

19 equations to describe the inter-population coupling (Jansen and Rit, 1995; Wendling et al., 20 2000): 
$\left\{\begin{array}{l}\dot{y}_{0}^{n}(t)=y_{5}^{n}(t) \\ \dot{y}_{5}^{n}(t)=A \cdot a \cdot S\left[y_{1}^{n}(t)-y_{2}^{n}(t)-y_{3}^{n}(t)\right]-2 a \cdot y_{5}^{n}(t)-a^{2} \cdot y_{0}^{n}(t) \\ \dot{y}_{1}^{n}(t)=y_{6}^{n}(t) \\ \dot{y}_{6}^{n}(t)=A \cdot a \cdot\left\{p^{n}(t)+C_{2} \cdot S\left[C_{1} \cdot y_{0}^{n}(t)\right]+\sum_{i \neq n} K_{i n} \cdot y_{10}^{i}(t)\right\}-2 a \cdot y_{6}^{n}(t)-a^{2} \cdot y_{1}^{n}(t) \\ \dot{y}_{2}^{n}(t)=y_{7}^{n}(t) \\ \dot{y}_{7}^{n}(t)=B \cdot b \cdot C_{4} \cdot S\left[C_{3} \cdot y_{0}^{n}(t)\right]-2 b \cdot y_{7}^{n}(t)-b^{2} \cdot y_{2}^{n}(t) \\ \dot{y}_{3}^{n}(t)=y_{8}^{n}(t) \\ \dot{y}_{8}^{n}(t)=G \cdot g \cdot C_{6} \cdot S\left[C_{5} \cdot y_{0}^{n}(t)-C_{7} \cdot y_{4}^{n}(t)\right]-2 g \cdot y_{8}^{n}(t)-g^{2} \cdot y_{3}^{n}(t) \\ \dot{y}_{4}^{n}(t)=y_{9}^{n}(t) \\ \dot{y}_{9}^{n}(t)=B \cdot b \cdot S\left[C_{3} \cdot y_{0}^{n}(t)\right]-2 b \cdot y_{9}^{n}(t)-b^{2} \cdot y_{4}^{n}(t) \\ \dot{y}_{10}^{n}(t)=y_{11}^{n}(t) \\ \dot{y}_{11}^{n}(t)=A \cdot a \cdot S\left[y_{1}^{n}(t)-y_{2}^{n}(t)-y_{3}^{n}(t)\right]-2 a \cdot y_{11}^{n}(t)-a^{2} \cdot y_{10}^{n}(t)\end{array}\right.$

2 where superscript $n$ denotes the population under consideration. For simplicity, the superscript $n$

3 is omitted for local parameters (A, B, G, a, b, g, and $\mathrm{C}_{1}$ to $\mathrm{C}_{7}$ ), although these parameters

4 depend on population $n$ and many vary from one population to another. The differential

5 equations were solved numerically using the fourth order Runge-Kunta method. At each

6 population $n$, the output signal $y^{n}(t)=y_{1}^{n}(t)-y_{2}^{n}(t)-y_{3}^{n}(t)$ is the summation of average

7 excitatory, slow inhibitory, and fast inhibitory postsynaptic potentials in the pyramidal cell

8 subpopulation. 


\section{References}

Abraham, K., Ajmone-Marsan, C.A., 1958. Patterns of cortical discharges and their relation to routine scalp electroencephalography. Electroencephalogr. Clin. Neurophysiol. 10(3): 447-461.

Alarcón, G., Binnie, C.D., Elwes, R.D., Polkey, C.E., 1995. Power spectrum and intracranial EEG patterns at seizure onset in partial epilepsy. Electroencephalogr. Clin. Neurophysiol. 94(5): 326337.

Allen, P.J., Fish, D.R., Smith, S.J., 1992. Very high-frequency rhythmic activity during SEEG suppression in frontal lobe epilepsy. Electroencephalogr. Clin. Neurophysiol. 82(2): 155-159.

Andrade-Valenca LP, Dubeau F, Mari F, Zelmann R, Gotman J (2011). Interictal scalp fast oscillations as a marker of the seizure onset zone. Neurology 77(6): 524-531.

Aubert, S., Wendling, F., Régis, J., McGonigal, A., Figarella-Branger, D., Peragut, J.C., Girard, N., Chauvel, P., Bartolomei, F., 2009. Local and remote epileptogenicity in focal cortical dysplasias and neurodevelopmental tumours. Brain 132: 3072-3086.

Bancaud, J., Talairach, J., Bonis, A., Schaub, C., Szikla, G., Morel, P., Bordas-Ferrer, M., 1965. "La stéréoélectroencéphalographie dans l'épilepsie: informations neurophysiopathologiques apportées par l'investigation fonctionnelle stereotaxique". Masson (Paris).

Bartolomei, F., Chauvel, P., Wendling, F., 2008. Epileptogenicity of brain structures in human temporal lobe epilepsy: a quantified study from intracerebral EEG. Brain 131: 1818-1830.

Bartolomei, F., Cosandier-Rimélé, D., McGonigal, A., Aubert, S., Régis, J., Gavaret, M., Wendling, F., Chauvel, P., 2010. From mesial temporal lobe to temporoperisylvian seizures: a quantified study of temporal lobe seizure networks. Epilepsia 51(10): 2147-2158.

Bartolomei, F., Wendling, F., Vignal, J.P., Kochen, S., Bellanger, J.J., Badier, J.M., Le Bouquin-Jeannes, R., Chauvel, P., 1999. Seizures of temporal lobe epilepsy: identification of subtypes by coherence analysis using stereo-electro-encephalography. Clin. Neurophysiol. 110(10): 1741-1754.

Bartos, M., Vida, I., Jonas, P., 2007. Synaptic mechanisms of synchronized gamma oscillations in inhibitory interneuron networks. Nat. Rev. Neurosci. 8(1): 45-56.

Benardo, L.S., 1994. Separate activation of fast and slow inhibitory postsynaptic potentials in rat neocortex in vitro. J. Physiol. 476(2): 203-215.

Bragin, A., Engel, J. Jr., Staba, R.J., 2010. High-frequency oscillations in epileptic brain. Curr. Opin. Neurol. 23(2): 151-156.

Cooper, R., Winter, A.L., Crow, H.J., Walter, W.G., 1965. Comparison of subcortical, cortical and scalp activity using chronically indwelling electrodes in man. Electroencephalogr. Clin. Neurophysiol. 18: 217-228. 
1 Cosandier-Rimélé, D., Badier, J.M., Chauvel, P., Wendling, F., 2007. A physiologically plausible spatiotemporal model for EEG signals recorded with intracerebral electrodes in human partial epilepsy. IEEE Trans. Biomed. Eng. 54(3): 380-388.

Cosandier-Rimélé, D., Merlet, I., Badier, J.M., Chauvel, P., Wendling, F., 2008. The neuronal sources of EEG: Modeling of simultaneous scalp and intracerebral recordings in epilepsy. Neuroimage 42(1): 135-146.

Deco, G., Jirsa, V.K., Robinson, P.A., Breakspear, M., Friston, K., 2008. The dynamic brain: from spiking neurons to neural masses and cortical fields. PLoS Comput. Biol. 4(8): e1000092.

DeLucchi, M.R., Garoutte, B., Aird, R.B., 1962. The scalp as an electroencephalographic averager. Electroencephalogr. Clin. Neurophysiol. 14: 191-196.

Demont-Guignard, S., Benquet, P., Gerber, U., Biraben, A., Martin, B., Wendlingm F., 2011. Distinct hyperexcitability mechanisms underlie fast ripples and epileptic spikes. Ann. Neurol. In press. (http://onlinelibrary.wiley.com/doi/10.1002/ana.22610/abstract).

Duckrow, R.B., Spencer, S.S., 1992. Regional coherence and the transfer of ictal activity during seizure onset in the medial temporal lobe. Electroencephalogr. Clin. Neurophysiol. 82(6): 415-422.

Fisher, R.S., Webber, W.R., Lesser, R.P., Arroyo, S., Uematsu, S., 1992. High-frequency EEG activity at the start of seizures. J. Clin. Neurophysiol. 9(3): 441-448.

Gastaut, H., Broughton, R., 1972. "Epileptic Seizures”. Springfield (USA).

Gnatkovsky, V., Librizzi, L., Trombin, F., de Curtis, M., 2008. Fast activity at seizure onset is mediated by inhibitory circuits in the entorhinal cortex in vitro. Ann. Neurol. 64(6): 674-686.

Gloor, P., 1985. Neuronal generators and the problem of localization in electroencephalography: application of volume conductor theory to electroencephalography. J. Clin. Neurophysiol. 2(4): 327-354.

Gonçalves, S.I., de Munck, J.C., Verbunt, J.P., Bijma, F., Heethaar, R.M., Lopes da Silva, F., 2003. In vivo measurement of the brain and skull resistivities using an EIT-based method and realistic models for the head. IEEE Trans. Biomed. Eng. 50(6): 754-767.

Gotman, J., 2010. High frequency oscillations: the new EEG frontier? Epilepsia 51(Suppl 1): 63-65.

Hämäläinen, M.S., Sarvas, J., 1989. Realistic conductivity geometry model of the human head for interpretation of neuromagnetic data. IEEE Trans. Biomed. Eng. 36(2): 165-171.

Jacobs, J., LeVan, P., Chander, R., Hall, J., Dubeau, F., Gotman, J., 2008. Interictal high-frequency oscillations $(80-500 \mathrm{~Hz})$ are an indicator of seizure onset areas independent of spikes in the human epileptic brain. Epilepsia. 49(11): 1893-1907. 
Jacobs, J., Zelmann, R., Jirsch, J., Chander, R., Dubeau, C.E., Gotman, J., 2009. High frequency oscillations $(80-500 \mathrm{~Hz})$ in the preictal period in patients with focal seizures. Epilepsia. 50(7): 1780-1792.

Jansen, B.H., Rit, V.G., 1995. Electroencephalogram and visual evoked potential generation in a 5 mathematical model of coupled cortical columns. Biol. Cybern. 73(4): 357-366.

Kobayashi K., Inoue T., Watanabe Y., Oka M., Endoh F., Yoshinaga H., Ohtsuka Y. (2009). Spectral

Kobayashi, K., Oka, M., Akiyama, T., Inoue, T., Abiru, K., Ogino, T., Yoshinaga, H., Ohtsuka, Y., Oka,

Lai, Y., van Drongelen, W., Ding, L., Hecox, K.E., Towle, V.L., Frim, D.M., He, B., 2005. Estimation of analysis of EEG gamma rhythms associated with tonic seizures in Lennox-Gastaut syndrome. Epilepsy Res. 86(1): 15-22. E., 2004. Very fast rhythmic activity on scalp EEG associated with epileptic spasms. Epilepsia 45(5): 488-496. in vivo human brain-to-skull conductivity ratio from simultaneous extra- and intra-cranial electrical potential recordings. Clin. Neurophysiol. 116(2): 456-465.

Le Van Quyen, M., Adam, C., Baulac, M., Martinerie, J., Varela, F.J., 1998. Nonlinear interdependencies of EEG signals in human intracranially recorded temporal lobe seizures. Brain Res. 792(1): 24-40.

Lee, S.A., Spencer, D.D., Spencer, S.S., 2000. Intracranial EEG seizure-onset patterns in neocortical epilepsy. Epilepsia 41(3): 297-307.

Malmivuo, J., Plonsey, R., 1995. "Bioelectromagnetism - Principles and Applications of Bioelectric and Biomagnetic Fields”. Oxford University Press (New York).

Markram, H., Toledo-Rodriguez, M., Wang, Y., Gupta, A., Silberberg, G., Wu, C., 2004. Interneurons of the neocortical inhibitory system. Nat. Rev. Neurosci. 5(10): 793-807.

Meijs, J.W., Weier, O.W., Peters, M.J., van Oosterom, A., 1989. On the numerical accuracy of the boundary element method. IEEE Trans. Biomed. Eng. 36(10): 1038-1049.

Molaee-Ardekani, B., Benquet, P., Bartolomei, F., Wendling, F., 2010. Computational modeling of highfrequency oscillations at the onset of neocortical partial seizures: From 'altered structure' to 'dysfunction'. Neuroimage 52(3): 1109-1122.

Oostendorp, T.F., Delbeke, J., Stegeman, D.F., 2000. The conductivity of the human skull: results of in vivo and in vitro measurements. IEEE Trans. Biomed. Eng. 47(11): 1487-1492.

Pacia, S.V., Ebersole, J.S., 1997. Intracranial EEG substrates of scalp ictal patterns from temporal lobe foci. Epilepsia 38(6): 642-654.

Pijn, J., Lopes da Silva, F., 1993. Propagation of electrical activity: nonlinear associations and time delays between EEG signals. In: Zschocke, S., Speckmann, E.J. (Eds), “Basic Mechanisms of the EEG”, Birkauser (Boston): 41-61. 
1 Plonsey, R., Heppner, D.B., 1967. Considerations of quasi-stationarity in electrophysiological systems. Bull. Math. Biophys. 29(4): 657-664.

Rush, S., Driscoll, D.A., 1968. Current distribution in the brain from surface electrodes. Anesth. Analg. 47(6): 717-723.

Somogyi, P., Tamás, G., Lujan, R., Buhl, E.H., 1998. Salient features of synaptic organisation in the cerebral cortex. Brain. Res. Rev. 26(2-3): 113-135.

Sporns, O., 2010. "Networks of the brain”. Cambridge, Mass.: MIT Press.

Tamás, G., Lorincz, A., Simon, A., Szabadics, J., 2003. Identified sources and targets of slow inhibition

Tao, J.X., Baldwin, M., Ray, A., Hawes-Ebersole, S., Ebersole, J., 2007. The impact of cerebral source area and synchrony on recording scalp electroencephalography ictal patterns. Epilepsia 48(11): 2167-2176.

Tao, J.X., Ray, A., Hawes-Ebersole, S., Ebersole, J., 2005. Intracranial EEG substrates of scalp EEG interictal spikes. Epilepsia 46(5): 669-676.

Traub, R.D., Duncan, R., Russell, A.J., Baldeweg, T., Tu, Y., Cunningham, M.O., Whittington, M.A., 2010. Spatiotemporal patterns of electrocorticographic very fast oscillations $(>80 \mathrm{~Hz})$ consistent with a network model based on electrical coupling between principal neurons. Epilepsia. 51(8): 1587-1597.

Traub, R.D., Whittington, M.A., Buhl, E.H., LeBeau, F.E., Bibbig, A., Boyd, S., Cross, H., Baldeweg, T., 2001. A possible role for gap junctions in generation of very fast EEG oscillations preceding the onset of, and perhaps initiating, seizures. Epilepsia 42(2): 153-170.

Ursino, M., Cona, F., Zavaglia, M., 2010. The generation of rhythms within a cortical region: analysis of a neural mass model. Neuroimage 52(3): 1080-1094.

Wendling, F., Bartolomei, F., Bellanger, J.J., Bourien, J., Chauvel, P., 2003. Epileptic fast intracerebral EEG activity: evidence for spatial decorrelation at seizure onset. Brain 126: 1449-1459.

Wendling, F., Bartolomei, F., Bellanger, J.J., Chauvel, P., 2001. Interpretation of interdependencies in epileptic signals using a macroscopic physiological model of the EEG. Clin. Neurophysiol. 112(7): 1201-1218.

Wendling, F., Bartolomei, F., Bellanger, J.J., Chauvel, P., 2002. Epileptic fast activity can be explained by a model of impaired GABAergic dendritic inhibition. Eur. J. Neurosci. 15(9): 1499-1508.

Wendling, F., Bellanger, J.J., Bartolomei, F., Chauvel, P., 2000. Relevance of nonlinear lumpedparameter models in the analysis of depth-EEG epileptic signals. Biol. Cybern. 83(4): 367-378. 
1 Wendling, F., Chauvel, P., 2008. Transition to ictal activity in temporal lobe epilepsy: insights from macroscopic models. In: Soltesz, I., Staley, K. (Eds), “Computational Neuroscience in Epilepsy”, Academic Press, pp. 356-386.

4 Wendling, F., Chauvel, P., Biraben, A., Bartolomei, F., 2010. From intracerebral EEG signals to brain connectivity: identification of epileptogenic networks in partial epilepsy. Front. Syst. Neurosci. 4: 154.

Wendling, F., Hernandez, A., Bellanger, J.J., Chauvel, P., Bartolomei, F., 2005. Interictal to ictal transition in human temporal lobe epilepsy: insights from a computational model of intracerebral EEG. J. Clin. Neurophysiol. 22(5): 343-356.

Wetjen, N.M., Marsh, W.R., Meyer, F.B., Cascino, G.D., So, E., Britton, J.W., Stead, S.M., Worrell, G.A., 2009. Intracranial electroencephalography seizure onset patterns and surgical outcomes in nonlesional extratemporal epilepsy. J. Neurosurg. 110(6): 1147-1152.

Whittington, M.A., Traub, R.D., Jefferys, J.G., 1995. Synchronized oscillations in interneuron networks driven by metabotropic glutamate receptor activation. Nature 373(6515): 612-615.

Whittington, M.A., Traub, R.D., Kopell, N., Ermentrout, B., Buhl, E.H., 2000. Inhibition-based rhythms: experimental and mathematical observations on network dynamics. Int. J. Psychophysiol. 38(3): 315-336

Worrell, G.A., So, E.L., Kazemi, J., O'Brien, T.J., Mosewich, R.K., Cascino, G.D., Meyer, F.B., Marsh, W.R., 2002. Focal ictal beta discharge on scalp EEG predicts excellent outcome of frontal lobe epilepsy surgery. Epilepsia 43(3): 277-282.

Wu, J.Y., Koh, S., Sankar, R., Mathern, G.W., 2008. Paroxysmal fast activity: an interictal scalp EEG marker of epileptogenesis in children. Epilepsy Res. 82(1):99-106. 


\section{Figure legends}
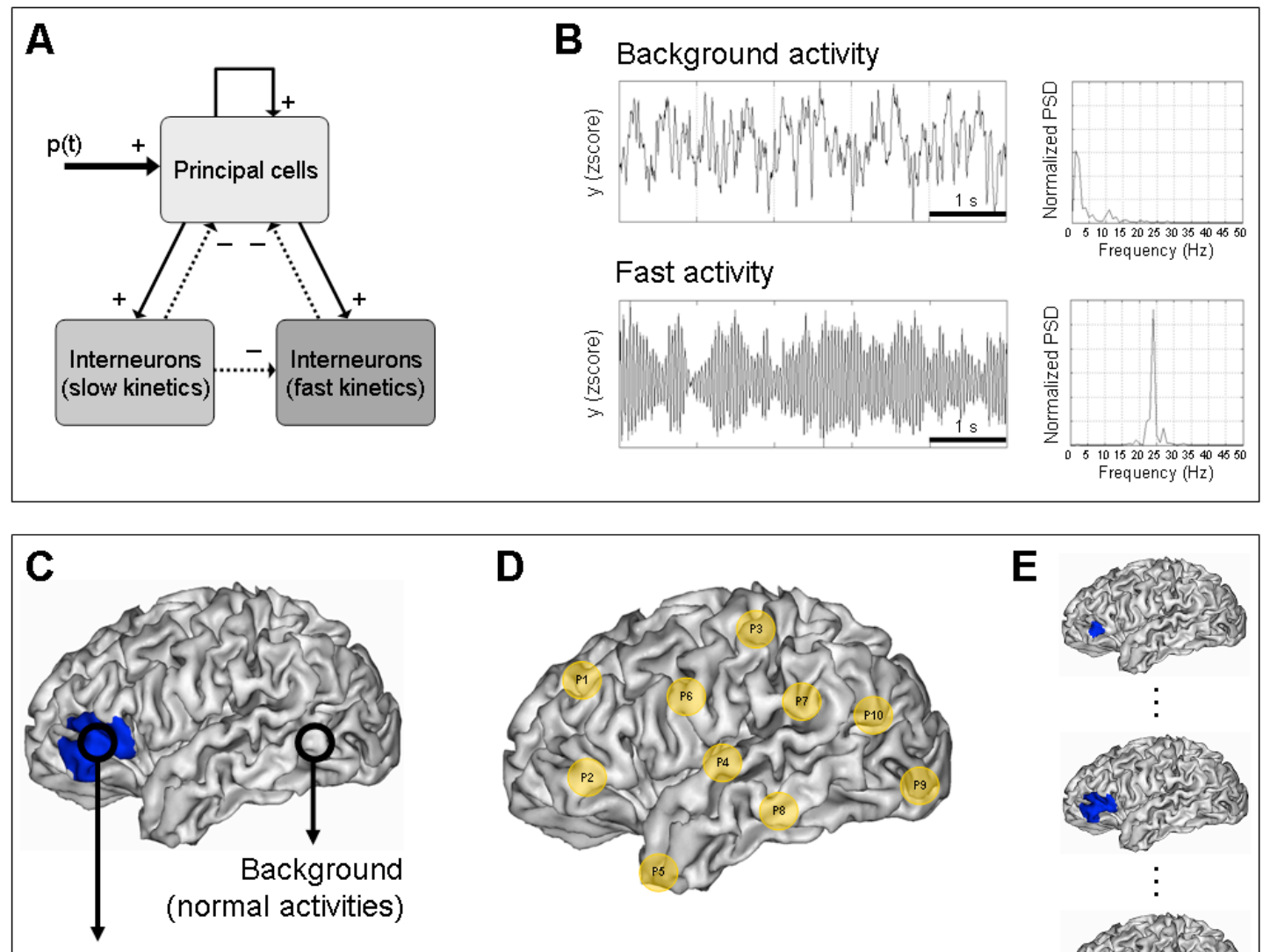

E

Epileptic source (fast activities)

3 Fig. 1: Computational model of EEG signals

4 Top: The neuronal population model. A) Structure of the model of a single population. It

5 comprises three interacting subpopulations of neurons: the excitatory pyramidal cells, inhibitory

6 interneurons with slow synaptic kinetics, and inhibitory interneurons with fast synaptic kinetics.

7 B) Typical signals produced by the model, representing a background activity and a fast activity,

8 and their power spectral density (PSD). The parameter values used to obtain these two signals

9 are given in the Appendix. Note that in figures, 'normalized' PSD is used (i.e. PSD divided by

10 the total power of the signal). Bottom: Computer simulations. In the model, EEG sources are

11 represented as dipole layers distributed over the cortical surface. C) A 'focal' epileptic source is

12 simulated by using a cortical patch inside which all current dipoles are assigned an epileptiform

13 time-course (fast activity), whereas all other dipoles, outside the patch, are assigned a 'normal'

14 time-course (background activity). D) Illustration of the ten epileptic patch locations (P1-P10), 
1 arbitrarily defined in the left hemisphere. E) Examples of three spatial extents for the epileptic

2 cortical patch (location P2): $\mathrm{S}=1 \mathrm{~cm}^{2}$ (top), $\mathrm{S}=10 \mathrm{~cm}^{2}$ (middle), and $\mathrm{S}=30 \mathrm{~cm}^{2}$ (bottom).
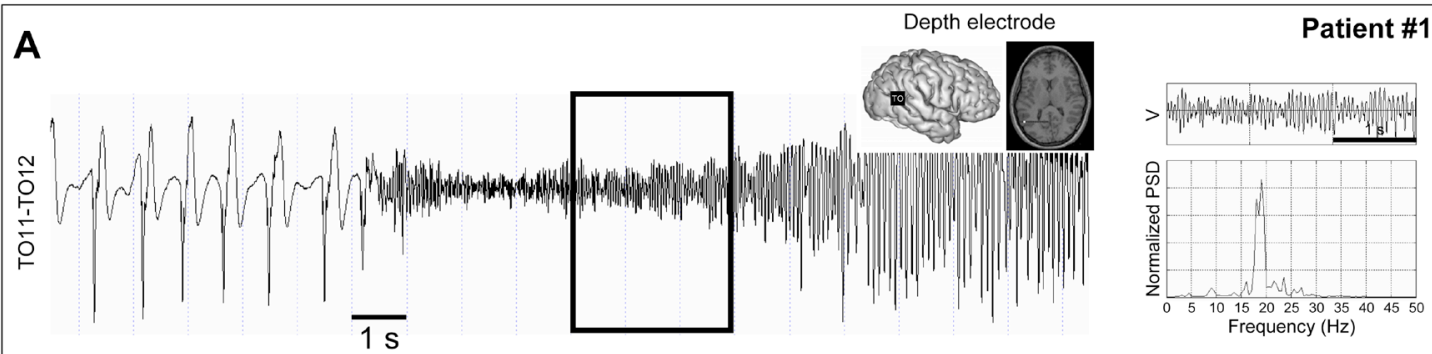

B

Scalp electrodes
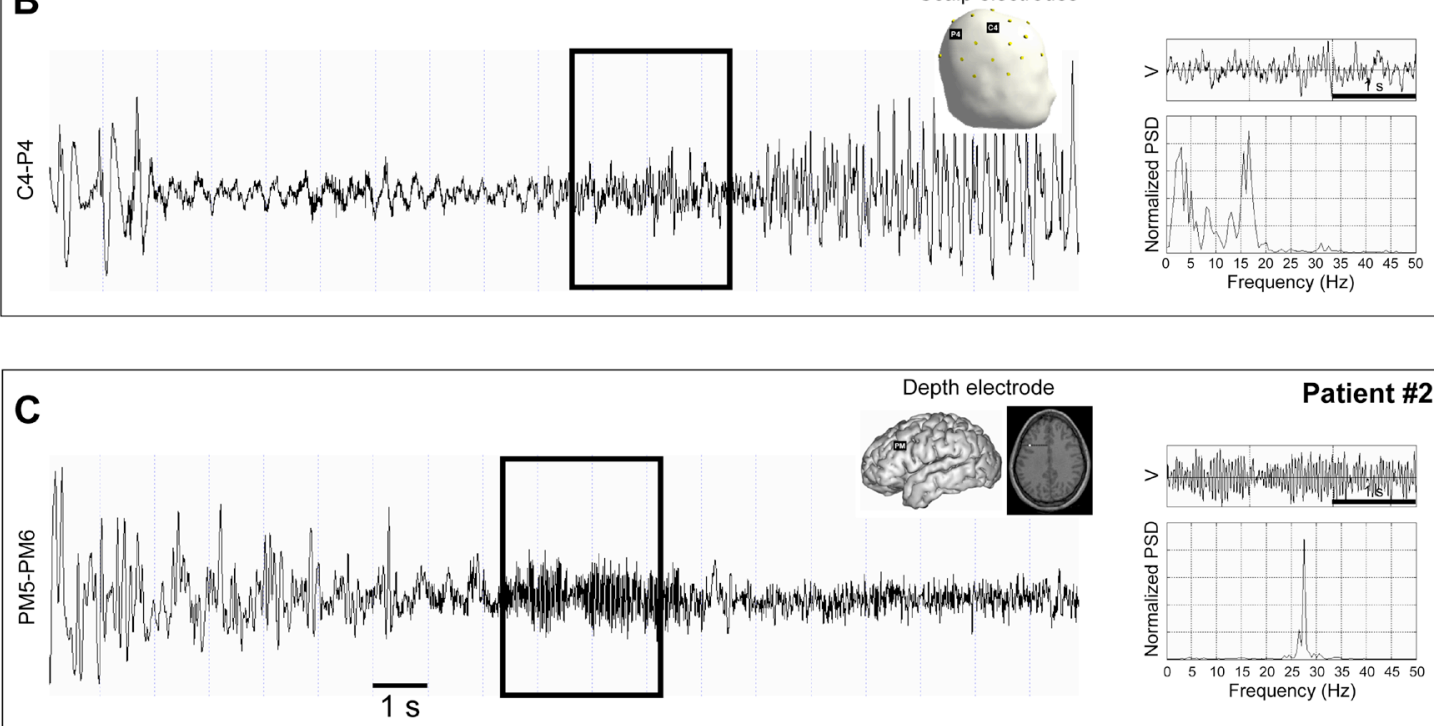

D
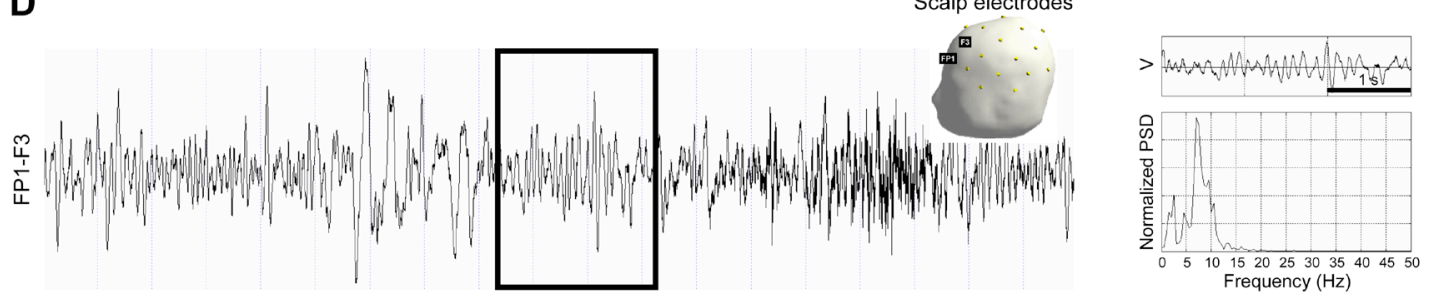

$5 \quad$ Fig. 2: Examples of real rapid discharges

6 Top: Case of a rapid discharge that is visible both in depth EEG and in scalp EEG. A) Example

7 of a depth EEG signal recorded from the temporo-occipital region (depth-electrode TO) in a

8 patient with drug-resistant partial neocortical epilepsy during the transition from interictal to

9 ictal activity, with a magnified view (3s segment) of the rapid discharge observed at seizure onset (indicated by the black box in the signal) and its PSD. B) Example of a scalp EEG signal

11 (C4-P4) recorded in the same patient during the transition to seizure, magnified view of the 
1 rapid discharge at seizure onset and its PSD. Bottom: Case of a rapid discharge that is visible in

2 depth EEG but not in scalp EEG. C) Example of a depth EEG signal recorded from the

3 premotor region (depth-electrode $\mathrm{PM}$ ) in a patient with drug-resistant partial neocortical

4 epilepsy during the interictal-to-ictal transition, magnified view ( $3 \mathrm{~s}$ segment) of the rapid

5 discharge at seizure onset and its PSD. D) Example of a scalp EEG signal (FP1-F3) recorded in

6 the same patient during the transition to seizure, with a magnified view at seizure onset (no

7 rapid discharge) and its PSD.

A

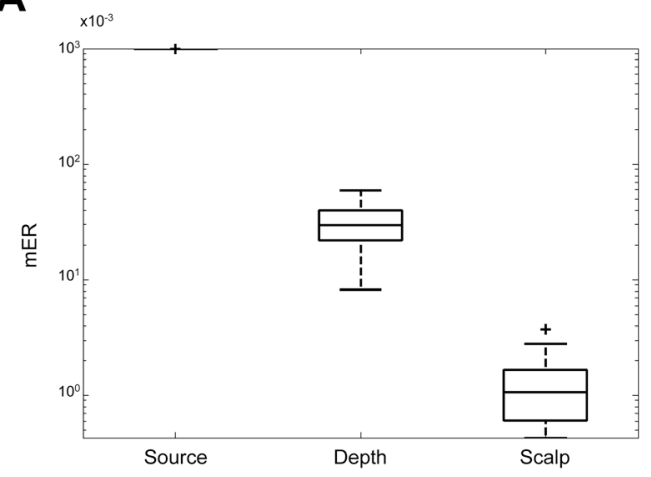

E

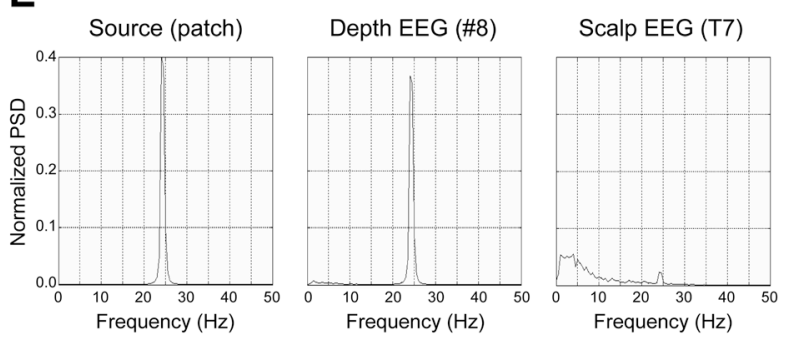

B

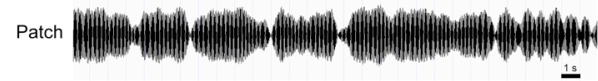

C

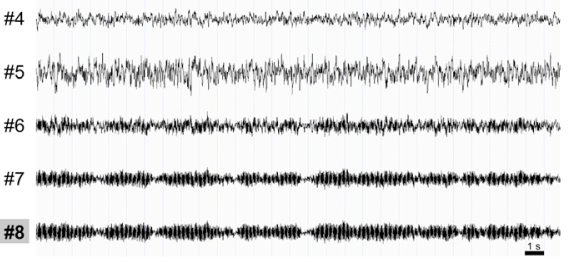

D

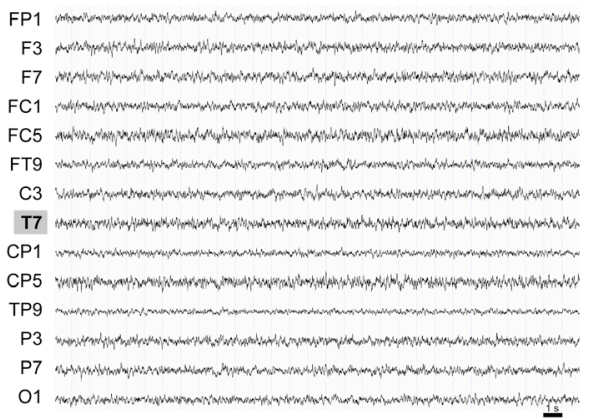

\section{Fig. 3: Simulation scenario 1 - Impact of the source-to-sensor distance}

1 A) Distributions of the mER values at 3 different recording sites: the cortical sources (patches), depth-electrode contacts and scalp electrodes. Boxplots represent the values obtained from the ten locations of the epileptic patch. Note the logarithmic scale of the y-axis. B) Example of a simulated signal for the activity of the epileptic source (patch location P4). C) Corresponding simulated depth EEG signals. D) Corresponding simulated scalp EEG signals. E) PSD of the source signal (patch), the signal from the depth-electrode contact that is closest to the epileptic patch (\#8), and the signal from the closest scalp electrode (T7). 
A

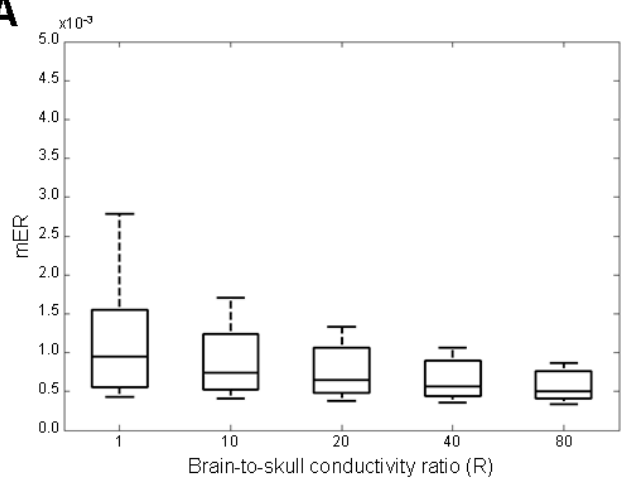

B
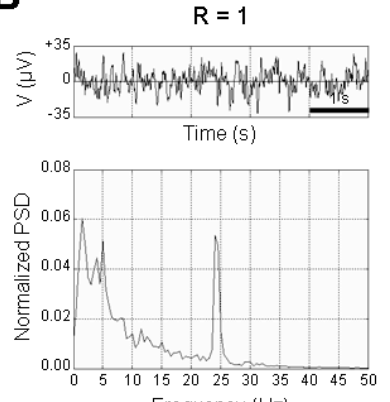

Frequency $(\mathrm{Hz})$
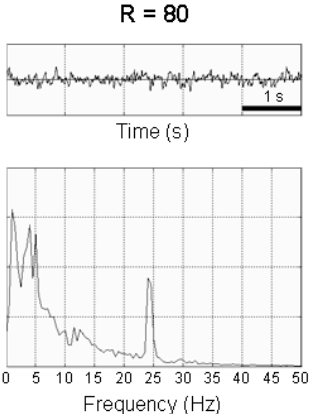

3 Fig. 4: Simulation scenario 2 - Impact of the skull conductivity

4 A) Distributions of the mER values for 5 values of brain-to-skull conductivity ratio (R). B)

5 Examples of simulated scalp EEG signals (5s segment) and their PSD for two values of R (the 6 minimum and maximum).

7

A
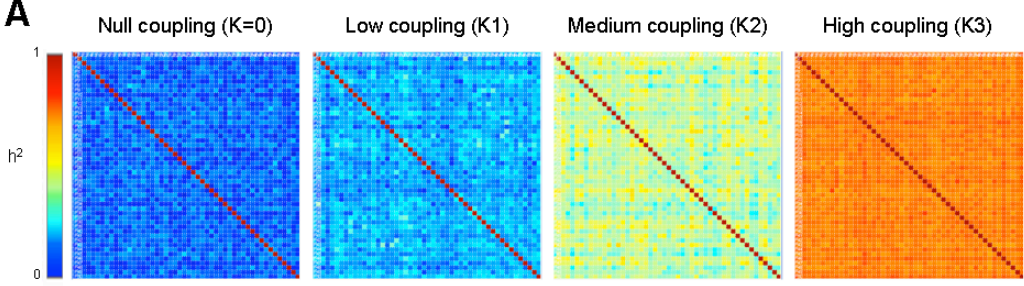

C Simulated depth EEG

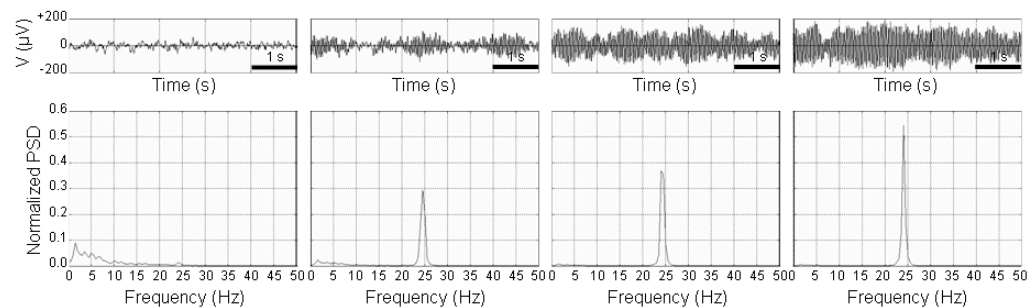

E Simulated scalp EEG
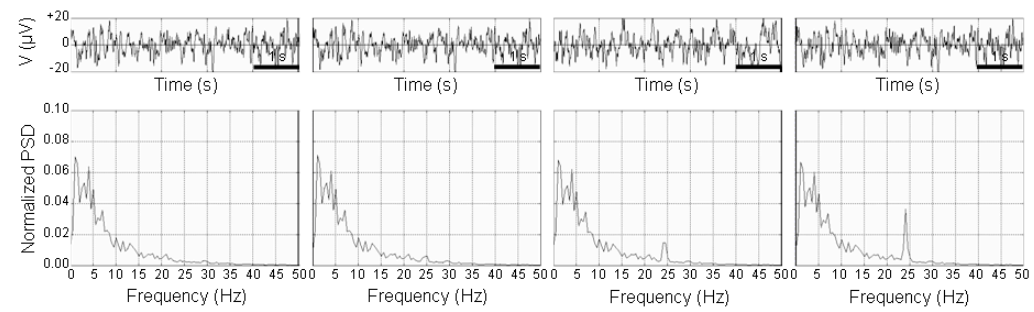

9 Fig. 5: Simulation scenario 3 - Impact of the source synchrony

10 Top: At the level of the cortical source. A) Matrices of correlation coefficients $\left(\mathrm{h}^{2}\right.$ indices)

11 calculated from the signals produced by a network of 50 neuronal populations, for 4 different
B

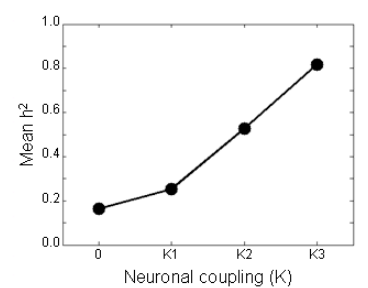

D

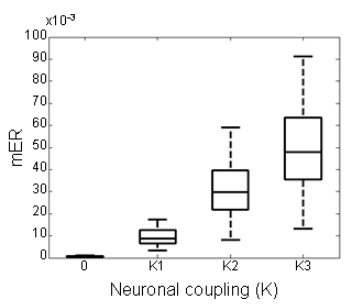

F

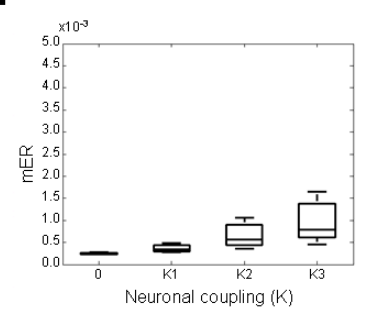


1 degrees of coupling (K) between the populations. B) Evolution of the mean $\mathrm{h}^{2}$ (average values

2 of the correlation matrices in A) as a function of K. Middle: At the level of depth electrodes. C)

3 Examples of simulated depth EEG signals (5s segment) and their PSD for the 4 values of K. D)

4 Distributions of the mER values for the ten epileptic patch locations. Bottom: At the level of 5 scalp electrodes. E) Examples of simulated scalp EEG signals and their PSD. F) Distributions 6 of the mER.

A

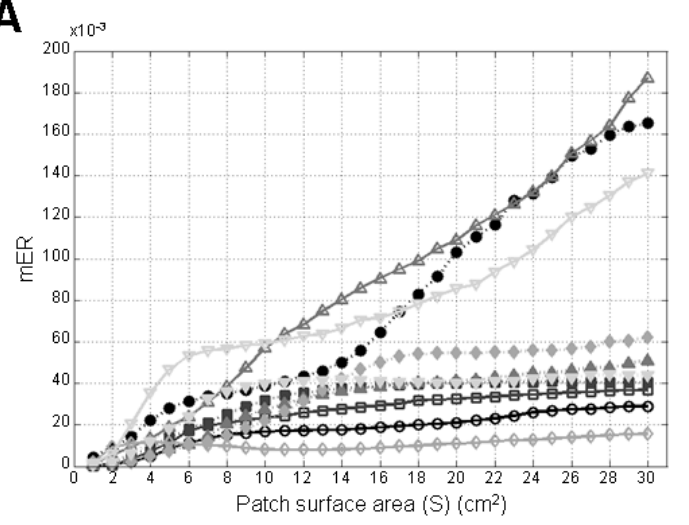

C Simulated depth EEG

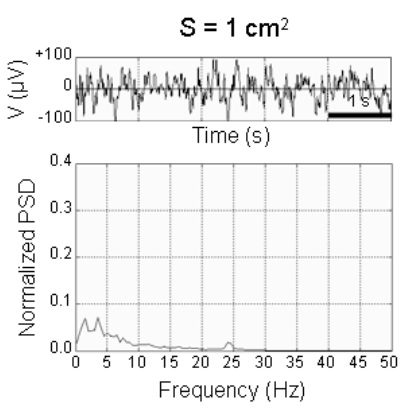

\section{Simulated scalp EEG}
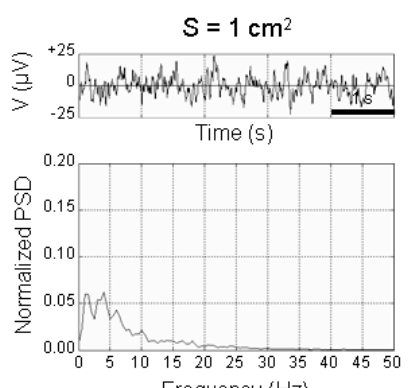

\section{B}

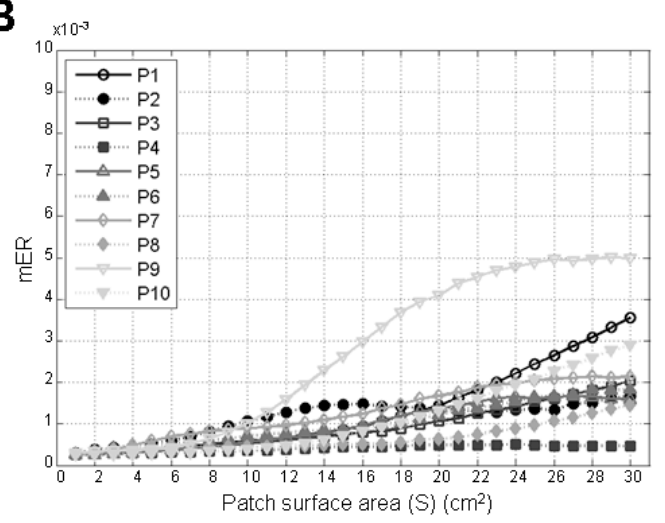

$S=10 \mathrm{~cm}^{2}$
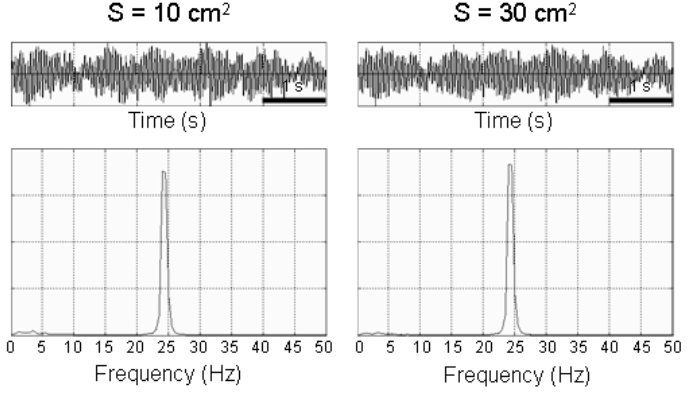

Frequency $(\mathrm{Hz})$
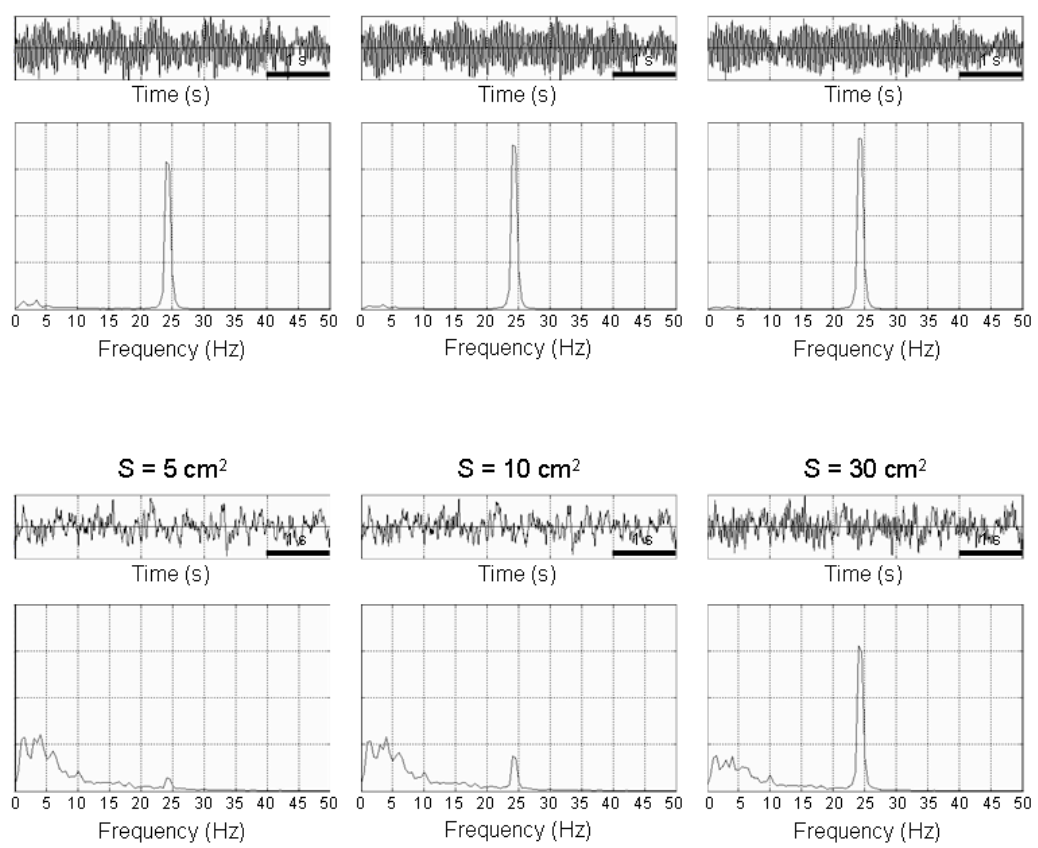

Fig. 6: Simulation scenario 4 - Impact of the source area

0 A) Evolution of the mER with respect to the surface area of the epileptic patch (S) in depth EEG,

1 for the ten patch locations. B) Evolution of the mER as a function of $\mathrm{S}$ in scalp EEG. Note the 
1 smaller range of the y-axis scaling. C) Examples of simulated depth EEG signals (5s segment)

2 and their PSD for 4 values of S. D) Examples of simulated scalp EEG signals and their PSD for

3 the same $\mathrm{S}$ values.

4

A

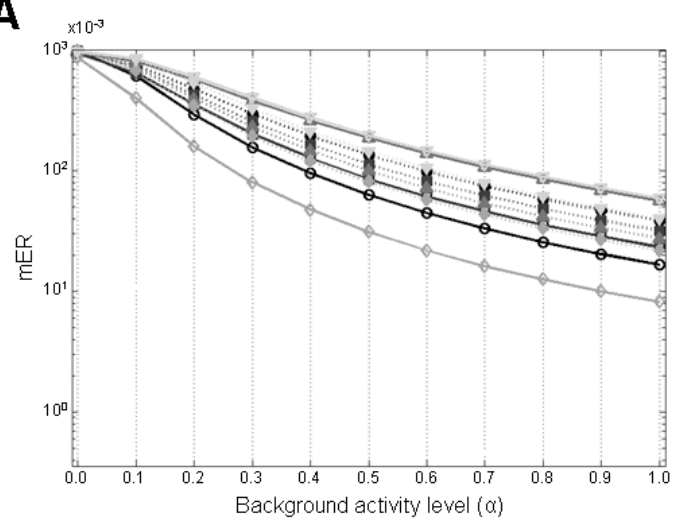

C Simulated depth EEG

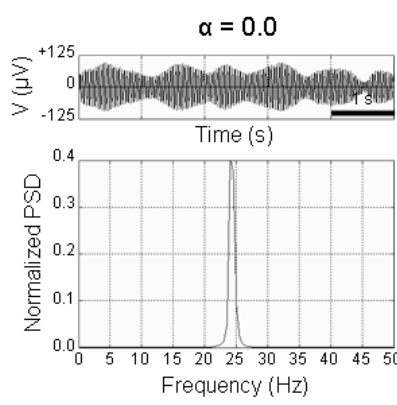

D Simulated scalp EEG
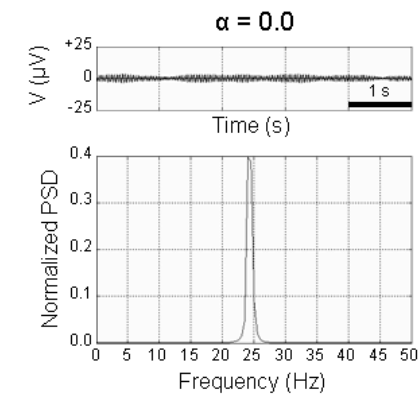
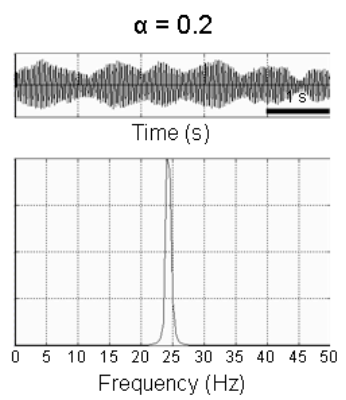

Frequency $(\mathrm{Hz})$
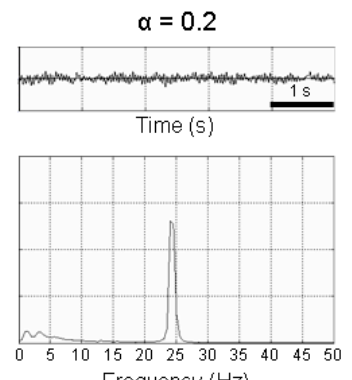

Frequency $(\mathrm{Hz})$
B

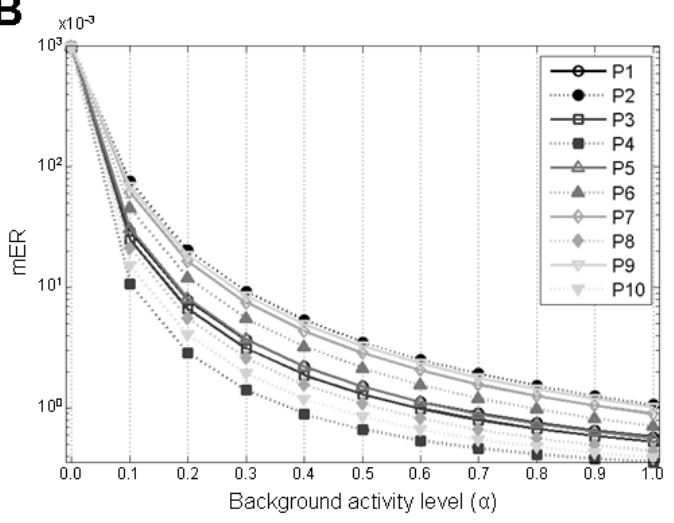

$\alpha=0.5$
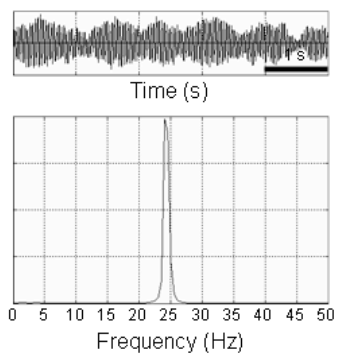

$\alpha=0.5$
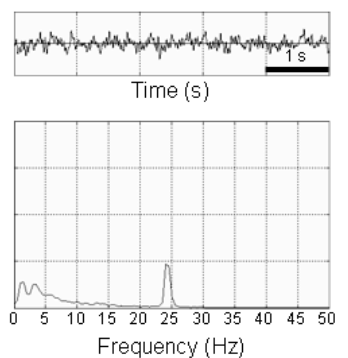

Frequency $(\mathrm{Hz})$

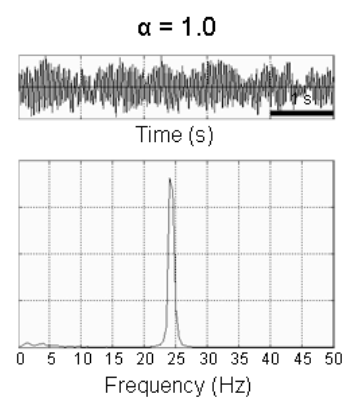

$\alpha=1.0$

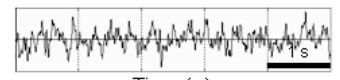
Time (s)

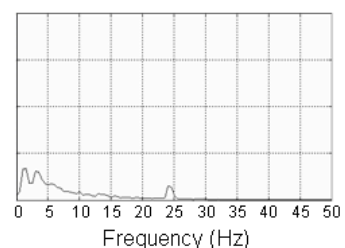

$6 \quad$ Fig. 7: Simulation scenario 5 - Impact of the background activity

7 A) Evolution of the mER with respect to the background activity level $(\alpha)$ in depth EEG, for the

8 ten epileptic patch locations. B) Evolution of the mER as a function of $\alpha$ in scalp EEG. Note the

$9 \log$ scale of the y-axis in both plots. C) Examples of simulated depth EEG signals (5s segment)

10 and their PSD for 4 values of $\alpha$. D) Examples of simulated scalp EEG signals and their PSD for

11 the same $\alpha$ values. 